\title{
The Dynamic Free Rider Problem: A Laboratory Study
}

\author{
By Marco Battaglini, Salvatore Nunnari, and Thomas R. Palfrey*
}

We report the results of an experiment that investigates free riding in the accumulation of durable public goods. We consider economies with reversibility, where contributions can be positive or negative; and economies with irreversibility, where contributions are nonnegative. Aggregate outcomes support the qualitative predictions of the Markov Perfect Equilibria (MPE) characterized in Battaglini, Nunnari, and Palfrey (2014): steady state levels of public good are lower with reversibility than irreversibility; accumulation is ineffciently slow; and the public good is under-provided in both regimes. On the other hand, public good levels are higher than MPE, and some evidence of history dependence is detected. (JEL C91, H41)

T

here is a vast literature addressing questions related to the provision of public goods in static environments. This includes hundreds of theoretical papers in the lineage initiated by Samuelson's (1954) seminal paper, presaged by the classical treatises on public finance by Wicksell and Lindahl. 1 It also includes hundreds of experimental papers based on one variation or another of Samuelson's theoretical model (Ledyard 1995). The typical motivating examples are national defense, public health, transportation infrastructure, pollution abatement, and so forth. What is striking is that essentially all economically important examples are public goods that take years to accumulate, provide streams of benefits over the long term, and require ongoing expenditures in order to improve or even maintain their levels. In other words, most public goods one can think of are durable goods and hence dynamics are an important component of their provision. In spite of this, remarkably little research has addressed the durable public goods problem from a dynamic perspective, especially in the experimental literature.

We are mainly interested in three questions: How serious is free riding in the provision of durable public goods? What new issues emerge from the dynamic nature

\footnotetext{
* Battaglini: Department of Economics, Cornell University, Uris Hall, Ithaca, NY 14850 (e-mail: battaglini@ cornell.edu); Nunnari: Department of Economics, Bocconi University, Via Roentgen 1, 20136 Milano, Italy, and Innocenzo Gasparini Institute for Economic Research (IGIER) (e-mail: salvatore.nunnari@ unibocconi.it); Palfrey: Division of the Humanities and Social Sciences, California Institute of Technology, MC 228-77, Pasadena, CA 91125 (trp@hss.caltech.edu). We are grateful for comments from a number of seminar audiences at universities and conferences. We thank Lydia Mechtenberg and Steven Matthews for valuable comments. Battaglini gratefully acknowledges financial support from NSF (SES-0418150) and the Alfred P. Sloan Foundation. Palfrey gratefully acknowledges financial support of grants from the National Science Foundation (SES-0962802, SES-1426560), the Gordon and Betty Moore Foundation (SES-1158), and the Russell Sage Foundation. Dustin Beckett and Anselm Rink provided valuable research assistance.

${ }^{\dagger}$ Go to http://dx.doi.org/10.1257/mic.20150126 to visit the article page for additional materials and author disclosure statement(s) or to comment in the online discussion forum.

${ }^{1}$ An excellent account of the development of the theory of public goods is Silvestre (2003).
} 
of the investment process? How do the answers to these questions depend on the degree to which investment decisions are reversible over time?

Dynamic free rider problems differ from static in subtle but important ways. In dynamic environments, we not only have the familiar free rider problem present in static public good provision, but also present is a second dynamic free rider phenomenon that further erodes incentives for efficient provision. In these games, strategies depend on the accumulated level of the public good, the state variable of the game: an increase in current investment by one agent typically triggers a reduction in future investment by all agents, in what is essentially a dynamic crowding-out effect. Such dynamic crowding out is especially severe if agents coordinate on stationary equilibria where strategies depend only on the accumulated level of the public good. On the other hand, the infinite horizon of the game generates a plethora of nonstationary equilibria that provide strategic opportunities to endogenously support cooperative outcomes using carrot-and-stick strategies. In principle, this could completely overcome both the static and the dynamic free rider problems. Thus, it is an open empirical question whether or not the free rider problem is exacerbated or ameliorated in the case of dynamic provision of durable public goods, as compared to one-shot public goods problems.

Dynamic free rider problems, moreover, offer a number of economically important predictions that cannot be assessed (or even stated) with static frameworks because they depend on the durability of the public good. First, regarding the storage technology, a public good is reversible if players can either increase it or decrease it transforming it back to private consumption; a public good is irreversible, if players cannot decrease it. Most investments are partially reversible, and the degree (or cost) of reversibility varies widely. ${ }^{2}$ What is the effect of irreversibility on contributions? Second, regarding the accumulation process, how are investment strategies going to depend on the state variable? If players use the state as a reference point, then the steady state may depend on the investments in the first periods: a good start with overinvestment (compared to the equilibrium level) may induce a permanent increase in the steady state. If agents instead are anchored to a given equilibrium steady state target, then players should be expected to correct "anomalous" contributions: overinvestment in the early periods should be corrected with underinvestment later on.

In this work, we make a first attempt to answer the questions raised above by studying the theoretical predictions of a simple dynamic public good game in a laboratory experiment. The economy we study has $n$ individuals. In each period, each individual is endowed with $w$ units of input that can be allocated between personal consumption and contribution to the stock of durable public good. Utility is linear in consumption of the private good and concave in the accumulated stock of the

\footnotetext{
${ }^{2}$ For example, the art collection at the Louvre, which took centuries to accumulate, could be sold off to private collectors and the proceeds distributed as transfer payments to the citizens of France. Cobblestone roads have been dug up and the stones used to build private dwellings. Military vehicles and aircraft can be (and have been) privatized and converted to civilian use. Publicly owned open space, even with conservation easements, are routinely converted to the private development of shopping malls, ski resorts, or new residential communities. Decades of sustainable management of fisheries, forests, or other replenishable resources can be rapidly reversed by over-harvesting or poaching.
} 
durable public good. Total payoffs for a player in the game are the discounted sum of utility over an infinite horizon of the game, where the discount factor is $\delta$. We characterize the efficient accumulation path as a function of $w, n$, and $\delta .{ }^{3}$ We study the Markov perfect equilibria of the game under two different assumptions about reversibility: full reversibility and irreversibility. We prove that investment is always higher in the irreversible case. We analyze the best subgame perfect equilibrium (a solution concept often used in applied work) of the two models and prove that the optimal investment strategies can be supported as an equilibrium with reversible investment but not with irreversible investment. The comparative static predictions implied by the two equilibrium concepts are completely opposed with respect to the effect of reversibility on investment: the Markov equilibrium predicts higher investments in a irreversible economy, the most efficient subgame perfect equilibrium predicts the opposite. These contrasting theoretical predictions are the basis for the main treatment in our experiment: reversibility versus irreversibility. We also have a secondary treatment dimension, which is the number of individuals in the game: we compare $n=3$ and $n=5$. Thus, the experiment has four different treatments depending on $n$ and whether investments are reversible.

The main comparative static prediction of the model is that in a Markov equilibrium there should be greater contributions and a higher equilibrium steady state level of public good in the irreversible investment economy (IIE) than in the reversible investment economy (RIE). The model also predicts small differences in public good levels as a function of $n$, with smaller groups accumulating slightly higher levels of public good (in the steady state for RIE; only on the convergence path for IIE). The data are consistent with the main predicted treatment effects: both in RIE and IIE the accumulation path is inefficiently slow and the public good under-provided; IIE induces significantly higher public good contributions than RIE. We do, however, observe some differences between the theoretical predictions and the data. In equilibrium, convergence should be monotonic. That is, the stock of public good should gradually increase over time until the steady state is reached, after which investment is zero. Instead, there is a tendency for initial overinvestment in the early periods, compared to the equilibrium investment levels. In the treatment with reversibility, this is followed by a significant reversal, i.e., negative investment, with the stock of public good gradually declining in the direction of the equilibrium steady state. After several periods of play, the stock of the public good is very close to the Markov equilibrium of the game. When disinvestment is not feasible, investment steadily decreases but it remains positive and the long-run level of the public good is significantly above the equilibrium steady state. Moreover, at least in a subset of periods, we observe a small but significant difference between groups of different sizes, with larger groups accumulating larger stocks.

In addition to the experiments described above, we propose a new methodology to test for Markovian behavior in equilibrium. The idea of the new experimental test consists in designing a one-period experiment where subjects' payoffs from

\footnotetext{
${ }^{3}$ To keep the experimental design simple, there is no depreciation, so at time $t$ the stock of the public good is simply the sum of individual investments across all periods up to time $t$. Battaglini, Nunnari, and Palfrey (2014) also characterize the efficient path and the equilibrium accumulation paths for arbitrary depreciation rates, $d \in[0,1]$.
} 
the public good are given by the equilibrium value function of the unique concave Markov perfect equilibrium of the game with reversibility. ${ }^{4}$ In this reduced-form version of the game, the individual incentives to contribute to the public good are exactly the same as in the fully dynamic game (under the assumption that subjects condition their strategies only on the public good stock), but there is no possibility to sustain a higher public good outcome through the nonstationary strategies that can arise in a repeated game. For many levels of the public good stock, we observe no difference in average contributions between this reduced form of the dynamic game and the fully dynamic game.

Our work is related to three distinct strands of research. First, naturally, is the experimental literature on public good provision in static environments. This literature has explored voluntary contributions under a variety of conditions. The early experiments focused primarily on free riding in environments where there was a dominant strategy for all individuals to contribute zero to the public good. Variations on these early dominant strategy public goods games have been conducted in the laboratory under many different assumptions about utility functions and technology, different subject pools, asymmetric endowments and preferences, different information conditions, different public good mechanisms, variable group sizes, and so forth. Many of these variations are discussed at length in Ledyard's (1995) comprehensive survey of the seminal work in this area. ${ }^{5}$ The dynamic environment we study is fundamentally different from the static environments studied in those papers. We have already mentioned some of these important differences and will discuss this issue in greater detail in Section $\mathrm{V}$, in the context of the results from our experiment.

The second literature to which our work is related consists in the work on sequential mechanisms for the provision of static public goods. Although in this literature players play a dynamic game, the purpose of the game is the determination of a one-shot provision of a discrete public good (Harrison and Hirschleifer 1989; Dorsey 1992; Duffy, Ochs, and Vesterlund 2007; Choi, Gale, and Kariv 2008; Diev and Hichri 2008; Noussair and Soo 2008; Choi et al. 2011)..$^{6}$ In the contribution games studied in these papers, agents have the opportunity to revise their initial contributions over time, and observe the cumulative level of contributions at each moment. Contrary to our setup, the public good does not provide any benefit until the game ends. Moreover, when payoffs from the public good are a continuous function of cumulative contributions, the unique equilibrium of these mechanisms is not different from the corresponding one-shot games (that is, no contribution). Only when a certain threshold guarantees a discrete benefit, agents might achieve the provision of this discrete public good, through history-dependent trigger strategies.

Third, our work is related to the emerging experimental literature on dynamic stochastic games in which a state variable provides a strategic link across periods. Early

\footnotetext{
${ }^{4}$ Cooper and Kühn (2014) use a two-period game where the period 2 payoffs are derived from the continuation value of an infinitely repeated version of the period 1 game. This preserves the strategic incentives and the multiplicity of equilibria of an infinitely repeated game in a finite horizon environment. Contrary to their approach, our one-period game has a unique equilibrium and is meant to eliminate the possibility to sustain more cooperative outcomes.

${ }^{5}$ See Chaudhuri (2011) and Vesterlund (2016) for more recent (and more selective) surveys.

${ }^{6}$ There are also public goods voluntary contribution experiments with reduced-form one-shot payoff functions that are motivated by common pool resource problems. See Ostrom (1999) for a survey.
} 
contributions are Noussair and Matheny (2000) and Lei and Noussair (2002) who experimentally study single agent dynamic optimization problems. Herr, Gardner, and Walker (1997) present a model of resource utilization in a finitely repeated environment in which players' actions have externalities on the preferences of current and future players. Battaglini and Palfrey (2012) test a dynamic model of pure redistribution (in which the state variable is the status quo distribution of resources and the amount of resources is constant over time). Battaglini, Nunnari, and Palfrey (2012) study the effect on investments of voting rules in a model of public good accumulation in which investment is chosen through a noncooperative bargaining process. To our knowledge Battaglini, Nunnari, and Palfrey (2010) is the first paper to present an experimental study of a durable public good game in which players make voluntary contributions. The results of that working paper are now incorporated in the present paper. ${ }^{7}$ Following this paper, Vespa (2016) has provided an alternative test of Markovian behavior in a game of resource exploitation similar to Herr, Gardner, and Walker (1997). ${ }^{8}$ The results of this paper confirm our finding that the Markov equilibrium is a good model of behavior in the laboratory. Interestingly, however, Vespa observes that the feasibility of cooperation with nonstationary strategies may depend on the complexity of the action space: cooperation may be possible with two actions, but not possible already with three. This may suggest that the Markov equilibrium can do well in environments like ours (with a continuum of actions) because players find it difficult to deal with the complexity of nonstationary strategies when the action space is nontrivial.

Finally, our work is more distantly related to the experimental literature on infinitely repeated prisoners' dilemma games. ${ }^{9}$ This literature has focused on identifying complicated history-dependent strategies and understanding the determinants of cooperation. To the contrary, we study a dynamic game with an endogenously evolving state variable ${ }^{10}$ and we focus on a different set of questions, namely, the comparison of outcomes under different storing technologies.

From a theoretical point of view, our work draws on Battaglini, Nunnari, and Palfrey (2014) who first characterized the equilibrium in the dynamic public good game that we study with and without reversibility. ${ }^{11}$ In our paper we use the characterization presented there as a basic prediction for the players' behavior in

\footnotetext{
${ }^{7}$ Battaglini, Nunnari, and Palfrey (2010) studied voluntary contribution only in the reversible case. The results presented in that working paper are now incorporated in the current expanded paper that includes also the irreversible case.

${ }^{8}$ Related experiments are presented by Saijo et al. (2009), who focus on an environment with static, state independent strategies, and Pevnitskaya and Ryvkin (2013), who focus on the effect of environmental context and termination uncertainty. The theoretical framework and experimental design of these papers do not investigate the effect of irreversibility on actions.

${ }^{9}$ See Dal Bó and Fréchette (2014) for a survey.

${ }^{10}$ Note also that, while in a prisoner's dilemma there is a dominant strategy, a one-period version of our game would have no dominant strategies.

${ }^{11}$ Previously, work on the reversible case was done in a framework of a linear differentiable game with quadratic preferences (Fershtman and Nitzan 1991 and Dockner and Long 1993, among others). Contribution games with irreversibility are studied in the literature on monotone games, see Matthews (2013) (and the references cited there) for a recent comprehensive analysis. These works, however, assume the players have a dominant strategy and it can be applied only to special cases that do not include our environment.
} 
the laboratory, integrating it with an analysis of other nonstationary equilibria for completeness.

The reminder of this paper is organized as follows. In Section I, we present the model and its solutions: the first best solution; the equilibrium when the public good is reversible; the equilibrium when the public good is irreversible; and the equilibrium predictions with nonstationary subgame perfect equilibria. In Section II, we describe the experimental design. Sections III and IV discusses the results of the experiment. Section V compares the results in our dynamic environment with the results of previous experiments on static public good games. Section VI concludes.

\section{The Model}

Here we describe a simplified version of the model in Battaglini, Nunnari, and Palfrey (2014), which we will use in our experimental design. Consider an economy with $n$ agents who interact for an infinite number of periods. There are two goods: a private good $x$ and a public good $g$. The level of consumption of the private good by agent $j$ in period $t$ is $x_{t}^{j}$, the level of the public good in period $t$ is $g_{t}$. The utility $U^{j}$ of agent $j$ can be written as

$$
U^{j}=\sum_{t=1}^{\infty} \delta^{t-1}\left[x_{t}^{j}+\alpha \sqrt{g_{t}}\right],
$$

where $\delta \in[0,1]$ is a common discount factor, and $\alpha>0$ is a constant. The private consumption good is nondurable, the public good is durable and does not depreciate between periods. Thus, if the level of public good at time $t-1$ is $g_{t-1}$ and the total investment in the public good is $I_{t}$, then the level of public good at time $t$ will be

$$
g_{t}=g_{t-1}+I_{t}
$$

It is convenient to distinguish the state variable at $t, g_{t-1}$, from the policy choice $g_{t}$. In the remainder, we denote $y_{t}=g_{t-1}+I_{t}$ as the new level of public good after an investment $I_{t}$ when the last period's level of the public good is $g_{t-1}$. The initial stock of public good is $g_{0} \geq 0$, exogenously given. Public policies are chosen as in the classic free rider problem. In each period, each agent $j$ is endowed with $w=W / n$ units of private good and chooses on its own how to allocate its endowment between an individual investment in the public good (which is shared by all agents) and private consumption, taking as given the strategies of the other agents. The key difference with respect to the static free rider problem is that the public good can be accumulated over time. The level of the state variable $g$, therefore, creates a dynamic linkage across policy making periods.

We consider two alternative economic environments. In a Reversible Investment Economy (RIE), the level of individual investment can be negative, with the constraint that $i_{t}^{j} \in\left[-g_{t} / n, W / n\right] \forall j$, where $i_{t}^{j}=W / n-x_{t}^{j}$ is the investment by 
agent $j .{ }^{12}$ In an Irreversible Investment Economy (IIE), an agent's investment cannot be negative and must satisfy $i_{t}^{j} \in[0, W / n] \forall j$.

The RIE corresponds to a situation in which the public investment can be scaled back in the future at no cost. An example can be an art collection or land for common use. The IIE corresponds to situations in which once the investment is done it cannot be undone. This seems the appropriate case for investments in public infrastructure (for example, a bridge or a road), the level of global warming, or less tangible investments like "social capital." In this environment, private consumption cannot be negative and the total economy-wide investment in the public good in any period is given by the sum of the agent investments.

\section{A. The Planner's Solution}

As a benchmark with which to compare the equilibrium allocations, we first analyze the sequence of public policies that would be chosen by a benevolent planner who maximizes the sum of utilities of the agents. This is the welfare optimum because the private good enters linearly in each agent's utility function.

Denote the planner's policy as $y_{P}(g)$ and consider first an economy with reversible investment. As shown by Battaglini, Nunnari, and Palfrey (2014), the objective function of the planner is continuous, strictly concave and differentiable, and a solution of its maximization problem exists and is unique. The optimal policies have an intuitive characterization. When the accumulated level of public good is low, the marginal benefit of investing in $g$ is high, and the planner finds it optimal to invest as much as possible: in this case $y_{P}(g)=W+g$ and $\sum_{j=1}^{n} x^{j}=0$. When $g$ is high, the planner will be able to reach the level of public good $y_{P}^{*}$ that solves the planner's unconstrained problem

$$
y_{P}^{*}=\left(\frac{\alpha n}{2(1-\delta)}\right)^{2}
$$

The investment function, therefore, has the following simple structure. For $g<y_{P}^{*}-W, y_{P}^{*}$ is not feasible: the planner invests everything and $y_{P}(g)=g+W$. For $g \geq y_{P}^{*}-W$, instead, investment stops at $y_{P}(g)=y_{P}^{*}$. This investment function implies that the planner's economy converges to the steady state $y_{P}^{o}=y_{P}^{*}$. In this steacly state, without loss of generality, we can set $x^{j}(g)$ $=\left(W+g-y^{P}(g)\right) / n \forall j \cdot{ }^{13}$

The planner's optimum for the IIE case is not very much different. The planner finds it optimal to invest all resources for $g \leq y_{P}^{*}-W$. For $g \in\left(y_{P}^{*}-W, y_{P}^{*}\right)$, the planner finds it optimal to stop investing at $y_{P}^{*}$, as before. For $g \geq y_{P}^{*}, y_{P}^{*}$ is not feasible, so it is optimal to invest 0 , and to set $y_{P}(g)=g$. This difference in the investment function for IIE, however, is essentially irrelevant for the optimal path

\footnotetext{
${ }^{12}$ This constraint guarantees that (out of equilibrium) the sum of reductions in $g$ can not be larger than the stock of $g$. The analysis would be similar if we allow each agent to withdraw up to $g$. In this case, however, we would have to assume a rationing rule in case the individuals withdraw more than $g$.

${ }^{13}$ Indeed, the planner is indifferent regarding the distribution of private consumption.
} 
and the steady state of the economy. Starting from any $g_{0}$ lower than the steady state $y_{P}^{*}$, levels of $g$ larger or equal than $y_{P}^{*}$ are impossible to reach, and the irreversibility constraint does not affect the optimal investment path.

\section{B. Reversible Investment Economies}

We first describe equilibrium behavior when the investment in the public good is reversible. We focus on continuous, symmetric Markov-perfect equilibria, where all agents use the same strategy, and these strategies are time-independent functions of the state, $g$. A strategy is a pair $(x(\cdot), i(\cdot))$ : where $x(g)$ is an agent's level of consumption and $i(g)$ is an agent's level of investment in the public good in state $g$. Associated with any equilibrium is a value function $v_{R}(g)$ which specifies the expected discounted future payoff to an agent when the state is $g$. The optimization problem for agent $j$ if the current level of public good is $g$, the agent's value function is $v_{R}(g)$, and other agents' investment strategies are given by $x_{R}(g)$, can be represented as

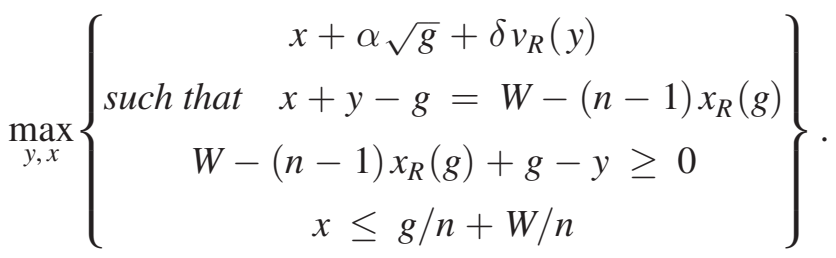

Contrary to the planner, agent $j$ cannot choose $y$ directly: it chooses only its level of private consumption and the level of its own contribution to the public investment. The agent, however, realizes that given the other agents' level of private consumption $(n-1) x_{R}(g)$, his/her investment ultimately determines $y$. It is therefore as if agent $j$ chooses $x$ and $y$, provided that he satisfies the feasibility constraints. The first constraint is the resource constraint: it requires that total resources, $W$, are equal to the sum of private consumption, $(n-1) x_{R}(g)+x$, plus the public investment, $y-g$. The second constraint requires that private consumption $x$ is nonnegative. The third constraint requires that no agent can reduce $y$ by more than his share $g / n$.

In a symmetric equilibrium, all agents consume the same fraction of resources, so agent $j$ takes as given that in state $g$ the other agents each consume

$$
x_{R}(g)=\frac{W+g-y_{R}(g)}{n},
$$

where $y_{R}(g)$ is the equilibrium level of investment in state $g$. Substituting the first constraint of (2) in the objective function, recognizing that agent $j$ takes the strategies of the other agents as given, and ignoring irrelevant constants, the agent's problem can be written as

$$
\max _{y}\left\{\begin{array}{c}
\alpha \sqrt{y}-y+\delta v_{R}(y) \\
y \leq \frac{W+g}{n}+\frac{n-1}{n} y_{R}(g), y \geq \frac{n-1}{n} y_{R}(g)
\end{array}\right\},
$$


where it should be noted that agent $j$ takes $y_{R}(g)$ as given. ${ }^{14}$ The objective function shows that an agent has a clear trade off: a dollar in investment produces a marginal benefit $\frac{\alpha}{2 \sqrt{g}}+\delta v_{R}^{\prime}(y)$, the marginal cost is -1 , a dollar less in private consumption. The first constraint shows that at the maximum the agent can increase the investment of the other players (i.e., $\left.\frac{n-1}{n} y_{R}(g)\right)$ by $\frac{W+g}{n}$. The second constraint makes clear that at most the agent can consume his endowment $W / n$ and his share of $g, g / n$.

We restrict attention to equilibria in which the objective function in (3) is strictly concave, and we refer to these equilibria as concave equilibria. Depending on the state $g$, the solution of (3) falls in one of two cases: the first case corresponds to the situation where the first constraint in (3) is binding, so all resources are devoted to investment in the public good. In this case, $x_{R}(g)=0, y_{R}(g)=W+g$, and investment by each agent is $i_{R}(g)=\frac{W}{n}$. In the second case, private consumption is positive, that is $x_{R}(g)>0$, and the solution is characterized by a unique public good level $y_{R}^{*}=\left(\frac{\alpha n}{2(n-\delta)}\right)^{2}$. In this second case, the investment by each agent is equal to $i_{R}(g)=\frac{1}{n}\left[y_{R}^{*}-g\right]$ and per capita private consumption is $x_{R}(g)=\frac{W+g-y_{R}^{*}}{n}>0$. The first case is possible if and only if $W \leq y_{R}^{*}-g_{R}$, that is, if $g$ is below some threshold $g_{R}$ defined by: $g_{R}=\max \left\{y_{R}^{*}-W, 0\right\}$. We summarize this in the following proposition, which also proves the existence of an equilibrium and its uniqueness when $v_{R}(g)$ is strictly concave.

PROPOSITION 1: In the game with reversible investment, a strictly concave equilibrium exists and it is unique. In this equilibrium, public investment is $y_{R}(g)=\min \left\{W+g, y_{R}^{*}\right\}$, where $y_{R}^{*}=\left(\frac{\alpha n}{2(n-\delta)}\right)^{2}<y_{P}^{*}$.

\section{PROOF:}

See Appendix A.

The public good function $y_{R}(g)$ is qualitatively similar to the corresponding planner's function $y_{P}(g)$. The main difference is that $y_{R}^{*}<y_{P}^{*}$ and $g_{R}<g_{P}$, so public good provision is typically smaller (and always smaller in the steady state). This is a dynamic version of the usual free rider problem associated with public good provision: each agent invests less than is socially optimal because he/she fails to fully internalize all agents' utilities. Part of the free rider problem can be seen from (3): in choosing investment, agents count only their marginal benefit, $u^{\prime}(y)+\delta v_{R}^{\prime}(y)$, rather than $n u^{\prime}(y)+\delta n v_{R}^{\prime}(y)$, but all the marginal costs $(-1)$. In this dynamic model, however, there is an additional effect that reduces incentives to invest, called the dynamic free rider problem. A marginal increase in $g$ has two effects. An immediate effect, corresponding to the increase in resources available in the following period: $g$. But there is also a delayed effect on next period's investment: the increase in $g$ triggers a reduction in the future investment of all the other agents through an increase in $x_{R}(g)$ : for any level of $g>g_{R}, y_{R}(g)$ will be kept at $y_{R}^{*}$.

\footnotetext{
${ }^{14}$ Since $y_{R}(g)$ is the equilibrium level of investment, in a symmetric equilibrium $(n-1) y_{R}(g) / n$ is the level of investment that agent $j$ expects from all the other agents, and that he/she takes as given in equilibrium.
} 
In a symmetric equilibrium, if agent $j$ increases the investment by one dollar, he will trigger a reduction in future investment by all agents by $1 / n$ dollars; the net value of the increase in $g$ for $j$ will be only $\delta / n$.

\section{Irreversible Investment Economies}

When the stock of the public good cannot be reduced, the optimization problem of an agent can be written like (2), but with an additional constraint: the individual level of investment cannot be negative or, in other words, each agent's private consumption cannot exceed his endowment, $x^{j}(g) \leq W / n$. Following similar steps as before, we can write the maximization problem faced by an agent as

$$
\max _{y}\left\{\begin{array}{c}
\alpha \sqrt{y}-y+\delta v_{I R}(y) \\
y \leq \frac{W+g}{n}+\frac{n-1}{n} y_{I R}(g), y \geq g+\frac{n-1}{n}\left(y_{I R}(g)-g\right)
\end{array}\right\},
$$

where the only difference with respect to (3) is the second constraint: the new level of public good cannot be lower than $g$ plus the investments from all the other agents.

As pointed out in Section IA, when public investments are efficient, irreversibility is irrelevant for the equilibrium allocation. The investment path chosen by the planner is unaffected because the planner's choice is time consistent: he never finds it optimal to increase $g$ if he plans to reduce it later. In the concave equilibrium characterized in the previous section, the investment function may be inefficient, but it is weakly increasing in the state. Agents invest until they reach a steady state, and then they stop. It may seem intuitive, therefore, that irreversibility is irrelevant in this case too, but this intuition is not correct. To the contrary, irreversibility destroys the concave equilibrium we characterized for reversible investment economies and induces the agents to significantly increase their investment, leading to a significantly higher unique steady state. Intuitively, the reason is that the agents no longer have to worry about the dynamic free rider problem: the irreversibility constraint creates a "commitment device" for the future; the agents know that $g$ cannot be reduced by the others (or their future selves).

PROPOSITION 2: In an economy with irreversible investment, a weakly concave equilibrium exists. Any weakly concave equilibrium, moreover, is associated to the same unique steady state equal to $y_{I R}^{*}=\left(\frac{\alpha}{2(1-\delta)}\right)^{2}$. This steady state level is strictly greater than $y_{R}^{*}$ and strictly smaller than $y_{P}^{*}$ for any $n>1$ and any $\delta \in[0,1)$.

\section{PROOF:}

See Appendix A.

The first part of Proposition 2 follows directly as a special case of proposition 1 in Battaglini, Nunnari, and Palfrey (2014), where it is established that the dynamic free rider game with irreversibility admits an equilibrium with standard concavity 
properties. The second part, uniqueness of the steady state, is established in Appendix A. In this steady state, the public good stock is strictly smaller than the one accumulated by a benevolent planner, but strictly higher than the one accumulated in the unique concave equilibrium of RIE. This steady state, $y_{I R}^{*}=\left(\frac{\alpha}{2(1-\delta)}\right)^{2}$, is exactly the same level that an agent alone would accumulate and it is independent of $n$.

The equilibrium selection based on Markov strategies therefore leads to a clear prediction that the irreversibility of investment will generate a higher level of investment in each period, as well as a higher steady state of the public good. The intuition for this is straightforward: the impossibility to convert today's investment back into private consumption at a future dates, eliminates worries about future agents' incentives to plunder the current public good investments. In other words, irreversibility mitigates the dynamic free rider problem. Moreover, the investment function in the equilibrium described in Proposition 2 is different than the one for the reversible investment case, where the agents would either find it optimal to invest everything, or just enough to reach the steady state. In contrast, in the irreversible investment case, the investment function increases gradually over time, ${ }^{15}$ and the steady state is reached only asymptotically.

\section{Cooperation Using Nonstationary Strategies}

We have restricted our attention to symmetric Markov perfect equilibria. However, the voluntary contribution game we study is an infinite horizon dynamic game with many subgame perfect equilibria. The Markovian assumption of stationary strategies is very restrictive and it is possible that some other equilibria can sustain more efficient outcomes through the use of history-dependent strategies that use punishments and rewards for past actions. As we show below, in economies with reversible investment, the optimal solution can indeed be supported as the outcome of a subgame perfect equilibrium.

PROPOSITION 3: There is a $\hat{\delta}_{R} \in[0,1)$, such that, for all $\delta>\hat{\delta}_{R}$, the efficient investment path characterized by the optimal solution is a Subgame Perfect Nash Equilibrium of the voluntary contribution game with reversible investment.

In Appendix A, we derive nonstationary strategies for the voluntary contribution game with reversible investment whose outcome is the efficient level of public good (the optimal solution), and show that these strategies are a subgame perfect Nash equilibrium. ${ }^{16}$

\footnotetext{
${ }^{15}$ This property of gradually increasing contributions in our model is reminiscent of a property of the repeated game equilibria found elsewhere in the literature (see, for example, Marx and Matthews 2000), but the intuition behind it is quite different. Here gradualism is needed in order to smooth out the value function of the Markov equilibrium at the steady state, while elsewhere gradualism follows from the non-Markov repeated game strategies that are used to enforce efficient equilibria.

${ }^{16}$ Our goal is to show that the optimal solution is the outcome of some subgame perfect Nash equilibria of the game. We do not claim that the strategies proposed in the proof of Proposition 3 are the best punishment schemes, and there may be different nonstationary strategies that work for lower $\delta$.
} 
The strategy for each agent is to allocate the optimal level of investment to public good production, $\left(y_{P}^{*}(g)-g\right) / n$, and to consume the remainder. A deviation from this investment behavior by any agent is punished by reversion to the unique concave Markov perfect equilibrium characterized in Section IB. This is a simple strategy that involves the harshest individually rational punishment for deviation from cooperation: whenever $g>y_{R}^{*}$ and a deviation is observed, the public good will revert to $y_{R}^{*}$ and it will stay at this level for all future periods.

When investment is irreversible, the efficient outcome cannot be sustained with strategies similar to the ones proposed above for environments with reversible investment. Matthews (2013) shows that, with discounting, no subgame perfect equilibrium of a general family of dynamic contribution games is efficient, in the sense of supporting the optimal public good stock in each period. In particular, that result applies to our environment, implying the following proposition as corollary.

PROPOSITION 4: There is no $\hat{\delta}_{I R} \in[0,1)$, such that, for all $\delta>\hat{\delta}_{I R}$, the optimal investment strategies are a Subgame Perfect Nash Equilibrium of the voluntary contribution game with irreversible investment.

The intuition behind Proposition 4 is that the potential for punishment is significantly dampened by the irreversibility constraint. Whenever $g>y_{I R}^{*}$ and a deviation is observed, agents cannot disinvest down to $y_{I R}^{*}$, and the harshest punishment is characterized by no investment and a constant stock in all periods following the first deviation.

Since many equilibria generally exist, a refinement is always needed for comparative statics or policy evaluation. It is standard practice in applied work to use as a solution concept the most efficient subgame perfect equilibrium (in our model the solution with the highest investment in $g$ ). The propositions presented above are important because they allow us to cleanly separate the time path of investment behavior implied by the Markov equilibrium discussed in the previous section from the time path of investment behavior in the best subgame perfect (non-Markov) equilibrium. Let $g_{t}^{M, R}$ and $g_{t}^{M, I R}$ denote the equilibrium stock of accumulated public good at time $t$ in the Markov equilibria discussed in the previous section with reversibility and irreversibility, respectively. Let $g_{t}^{S, R}$ and $g_{t}^{S, I R}$ be the corresponding stock of accumulated public good observable at time $t$ in the best subgame perfect equilibrium. We have:

COROLLARY 1: There is a $\delta^{*} \in[0,1)$, such that, for $\delta>\delta^{*}$, we have:

- $g_{t}^{M, R}<g_{t}^{M, I R}$ on the equilibrium path.

- $g_{t}^{S, I R} \leq g_{t}^{S, R}$ on the equilibrium path.

The first bullet point can be established using Propositions 1 and 2, while the second bullet point follows from Propositions 3 and 4. Corollary 1 establishes that the comparative static predictions implied by the two different equilibrium concepts (Markov versus best SPE) are completely opposed with respect to the effect of reversibility versus irreversibility on investment. This theoretical insight thus 
provides two starkly opposite predictions about efficiency that will be useful for interpreting the results of the experiment.

\section{Experimental Design}

The experiments were conducted at the Social Science Experimental Laboratory (SSEL) using students from the California Institute of Technology, and at the Columbia Experimental Laboratory for the Social Sciences (CELSS) using students from Columbia University. Subjects were recruited from pools of volunteer subjects, maintained by SSEL and CELSS. Ten sessions were run, using a total of 129 subjects. No subject participated in more than one session. Half of the sessions were for Reversible Investment Economies and half for Irreversible Investment Economies. Six sessions were conducted using three person groups, and four with five person groups. In all sessions the discount factor was $\delta=0.75$, and the multiplier of the current-period payoff from the public good was $\alpha=4$, that is, $u(g)=4 \sqrt{g}$. In the 3 person groups, we used the parameters $W=60$, while in the 5 person groups $W=80$. It is useful to emphasize that, as proven in the previous sections, given these parameters the steady state is uniquely defined both for the RIE and IIE game and for all treatments.

Discounted payoffs were induced by a random termination rule by rolling a die after each period in front of the room, with the outcome determining whether the game continued to another period (with probability 0.75 ) or was terminated (with probability 0.25 ). The $n=5$ sessions were conducted with 15 subjects, divided into 3 groups of 5 members each. The $n=3$ sessions were conducted with 12 subjects, divided into 4 groups of 3 members each. ${ }^{17}$ Groups stayed the same throughout the periods of a given match, and subjects were randomly rematched into groups between matches. A match consisted of one multi-period play of the game, which continued until one of the die rolls eventually ended the match. As a result, different matches lasted for different lengths (that is, for a different number of periods). In all sessions, subjects interacted for ten matches. Table 1 summarizes the design and the theoretical properties of the equilibrium for the four treatments.

Before the first match, instructions ${ }^{18}$ were read aloud, followed by a practice match and a comprehension quiz to verify that subjects understood the details of the environment including how to compute payoffs. The current period's payoffs from the public good stock (called project size in the experiment) was displayed graphically, with stock of public good on the horizontal axis and the payoff on the vertical axis. Subjects could click anywhere on the curve and the payoff for that level of public good appeared on the screen. Subjects received information about the total investment in the public good as well as about the individual investments of other subjects in their group, at the end of each period. At the end of the last match each subject was paid privately in cash the sum of his or her earnings over all matches

\footnotetext{
${ }^{17}$ One of the $N=3$ sessions used nine subjects.

${ }^{18}$ Sample instructions are reported in Appendix C. Subject decisions, interactions, and feedback were implemented in a computer network using the open source interactive game software, Multistage (http://software.ssel. caltech.edu/).
} 
Table 1-Experimental Design, Equilibrium, and Planner Steady States

\begin{tabular}{lccccccc}
\hline \hline Treatment & $n$ & $W$ & Sessions & Subjects & Groups & $y_{M P E}^{*}$ & $y_{P}^{*}$ \\
\hline RIE & 3 & 60 & 3 & 33 & 110 & 7.11 & 576 \\
RIE & 5 & 80 & 2 & 30 & 60 & 5.54 & 1,600 \\
IIE & 3 & 60 & 3 & 36 & 120 & 64 & 576 \\
IIE & 5 & 80 & 2 & 30 & 60 & 64 & 1,600 \\
\hline
\end{tabular}

Table 2-Long-Run Stock of Public Good, Theory versus Results

\begin{tabular}{lcccc}
\hline \hline Treatment & $n$ & $y_{m d n}^{L R}$ & $y_{M P}^{L R}$ & $y_{P}^{L R}$ \\
\hline Reversible investment (RIE) & 3 & 16 & 7.11 & 576 \\
Reversible investment (RIE) & 5 & 26 & 5.54 & 800 \\
Irreversible investment (IIE) & 3 & 306 & 43.64 & 576 \\
Irreversible investment (IIE) & 5 & 350 & 42.20 & 800 \\
\hline
\end{tabular}

plus a show-up fee of $\$ 10$. Earnings ranged from approximately $\$ 20$ to $\$ 50$, with sessions lasting between one and two hours. There was considerable range in the earnings and length across sessions because of the random stopping rule.

\section{Experimental Results}

\section{A. Public Good Outcomes}

We start the analysis of the experimental results by looking at the long-run stock of public good by treatment. We consider as the long-run stock of public good, the stock reached by a group after ten periods of play. ${ }^{19}$ Table 2 compares the theoretical and observed levels of public good by treatment. In order to aggregate across groups, we use the median level of the public good from all groups in a given treatment in periods 9-11 $\left(y_{m d n}^{L R}\right)$. Similar results hold if we use a different subset of periods around ten, or look at the mean or other measures of central tendency. ${ }^{20}$ We compare this to the stock predicted by the Markov perfect equilibrium of the game after ten periods $\left(\frac{I R}{y_{M I P}}\right)$, and to the stock accumulated in the optimal solution after ten periods $\left(y_{P}^{L R}\right) .21$

How do groups arrive at these period 10 stocks of public good? Figures 1 and 2 give us a richer picture, showing the time series of the stock of public good by treatment. ${ }^{22}$ The horizontal axis is the time period and the vertical axis is the stock

\footnotetext{
${ }^{19}$ In the experiment, the length of a match is stochastic and determined by the roll of a die. No match lasted longer than 17 periods and we have very few observations for periods 11-17. Detailed information on the number of observations for each period is reported in Tables B1 and B2 in Appendix B.

${ }^{20}$ In Appendix B, we report averages, medians, and standard errors of the stock of the public good by period for each treatment. When discussing long-run outcomes, we pool together periods 9-11 to consider a larger sample. The median in Table 2 is computed from 17 observations for RIE with $n=3,15$ observations for RIE with $n=5$, 68 observations for IIE with $n=3$, and 32 observations for IIE with $n=5$. In spite of the lower number of observations, results are unchanged when we consider only period 10 stocks as long run outcomes.

${ }^{21}$ Note that the median stock predicted for periods 9-11 is the stock predicted for period 10 .

${ }^{22}$ These and subsequent figures show data from the first ten periods. Data from later periods (11-13 for RIE and 11-17 for IIE) are excluded from the graphs because there were so few observations. The data from later periods are reported in Appendix B and included in all the statistical analyses.
} 
Panel A. $n=3$
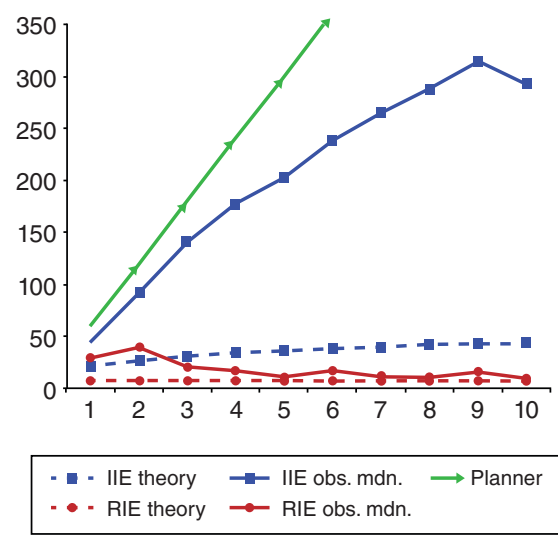

Panel B. $n=5$

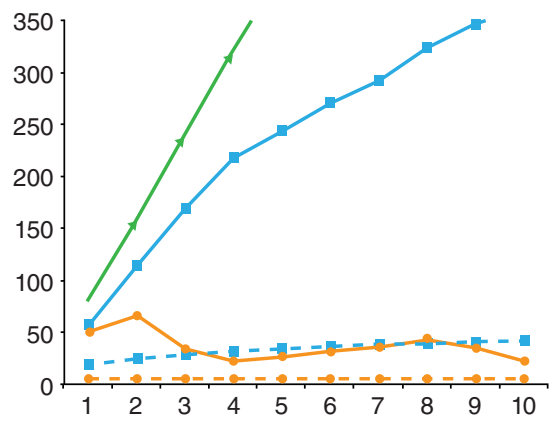

$=-$ IIE theory $\quad \because$ IIE obs. mdn. $\longrightarrow$ Planner
$-*$ RIE theory $\quad \longrightarrow$ RIE obs. mdn.

Figure 1. Median Time Paths of the Stock of $g$, RIE versus IIE

Panel A. $n=3$

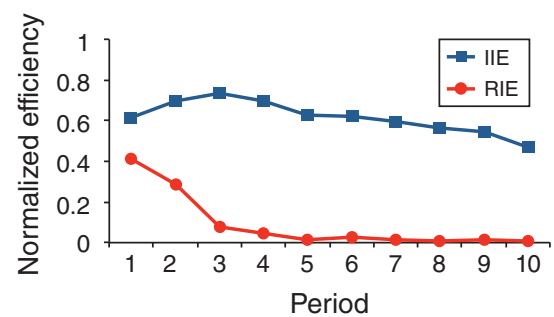

Panel B. $n=5$

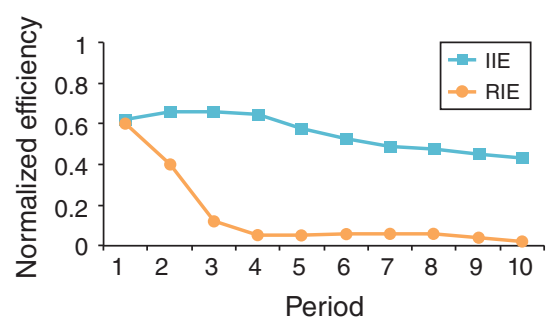

Figure 2. Median Time Paths of Normalized Efficiency, RIE versus IIE

of the public good. We use the median level of the public good from all groups in a given treatment. Superimposed on the graphs are the theoretical time paths corresponding to the Markov perfect equilibria (represented with dashed lines) and to the optimal solution. Table 2, Figure 1, and Figure 2 exhibit several systematic regularities, which we discuss below in comparison with the theoretical time paths.

Finding 1: Irreversible investment leads to higher public good production than reversible investment. According to Wilcoxon-Mann-Whitney tests, ${ }^{23}$ the stock of public good is significantly lower in RIE than in IIE in every single period. This difference is statistically significant at the 1 percent level $(p<0.01)$ for periods 1-10 for both group sizes (see Table 3, columns 1 and 2). Not only are the differences statistically significant, but they are large in magnitude. The median stock of

\footnotetext{
${ }^{23}$ Unless otherwise noted, our significance tests are based on Wilcoxon-Mann-Whitney tests. The null hypothesis of a Wilcoxon-Mann-Whitney test is that the underlying distributions of the two samples are the same. We are treating as unit of observation a single group. The results are unchanged if we use $t$-tests for differences in means (see Table B5 n Appendix B). We take into account the panel structure of the data by clustering the standard errors of these tests by groups composed of the same subjects.
} 
TABle 3-Period-By-Period $p$-VAlues of Wilcoxon-Mann-Whitney Tests for Public Good STOCKS

\begin{tabular}{ccccc}
\hline \hline Period & RIE3/IIE3 & RIE5/IIE5 & RIE3/RIE5 & IIE3/IIE5 \\
\hline 1 & 0.000 & 0.005 & 0.000 & 0.000 \\
2 & 0.000 & 0.000 & 0.001 & 0.000 \\
3 & 0.000 & 0.000 & 0.061 & 0.000 \\
4 & 0.000 & 0.000 & 0.057 & 0.003 \\
5 & 0.000 & 0.000 & 0.049 & 0.010 \\
6 & 0.000 & 0.000 & 0.009 & 0.144 \\
7 & 0.000 & 0.000 & 0.259 & 0.284 \\
8 & 0.000 & 0.000 & 0.000 & 0.580 \\
9 & 0.000 & 0.001 & 0.047 & 0.668 \\
10 & 0.006 & 0.001 & 0.627 & 0.313 \\
\hline
\end{tabular}

public good is many times greater in the IIE treatment than in the RIE treatment, averaged across all periods for which we have data (154 in IIE versus 24 in RIE for $n=3 ; 155$ in IIE versus 43 in RIE for $n=5$ ). The difference between the two treatments is relatively small in the initial periods, but it increases sharply as more periods are played. By period 10, the difference is very large (293.5 versus 10 for $n=3$ and 367 versus 22 for $n=5$ ).

Finding 2: Both reversible and irreversible investment lead to significantly inefficient long-run public good levels. The optimal steady state is $y^{*}=576$ for $n=3$ and $y^{*}=1,600$ for $n=5$, and the optimal investment policy is the fastest approach: invest $W$ in every period until $y^{*}$ is achieved. After 10 periods, the median stock of public good achieved with the optimal investment trajectory is $\mathbf{5 7 6}$ with $n=3$ and 800 with $n=5$. In the experiments, the median stock of public good levels out at about $12(n=3)$ or $33(n=5)$ under reversible investment economies, while it keeps growing, but at an inefficiently slow pace, under irreversible investment. The median stock in periods $9-11$ is 16 in RIE with $n=3,26$ in RIE with $n=5,306$ in IIE with $n=3$, and 350 in IIE with $n=5$. In all treatments, the average stock of public good in periods $9-11$ is significantly smaller than the level predicted by the optimal solution (the level attainable investing $W$ each period) according to the results of a $t$-test on the equality of means with standard errors clustered at the group level $(p<0.01)$.

Finding 3: In IIE, the efficiency of long-run public good levels is midway between the planner solution and the prediction of the MPE. In RIE, efficiency converges close to MPE steady state. For each period $t$, we define a normalized efficiency measure as $E^{t}=\frac{\left(y^{t}-y_{M P}^{t}\right)}{\left(y_{P}^{t}-y_{M P}^{t}\right)}$, where $y_{M P}^{t}$, the public good stock predicted by the MPE, is the lower bound for efficiency, $y_{P}^{t}$, the public good stock in the planner solution, is the upper bound, and $y^{t}$ is the median observed public good stock in period $t$. Figure 2 shows the evolution of $E^{t}$ over time for the two treatments. In periods 9-11, $E^{t}$ ranges between 0.02 and 0.04 with reversible investment and ranges between 0.39 and 0.53 with irreversible investment. We highlight two results. First, 
IIE is several orders of magnitude more efficient than RIE. Second, RIE efficiency is essentially at the lower bound in the long run, indicating convergence to the prediction of the MPE, while IIE efficiency is always very high. ${ }^{24}$

From Proposition 3, we know that, for the parameters of the experiment, almost efficient levels of the public good can be supported as the outcome of the RIE game using nonstationary subgame perfect strategies. ${ }^{25}$ In the IIE games, on the other hand, the optimal solution cannot be supported by any subgame perfect equilibrium with nonstationary strategies when there is discounting. This is in stark contrast with the unique Markov perfect equilibria derived in Sections IB and IC, which imply the opposite comparative static: the long-run level of the public good is predicted to be ten times as large with irreversible investment than with reversible investment.

The analysis of the public good outcomes suggests that the predictions of the Markov perfect equilibrium are substantially more accurate than the prediction of the "best" subgame perfect equilibrium (that is the Pareto superior equilibrium from the point of view of the agents), in terms of both the long-run stock of public good in RIE and the effect of reversibility. On the other hand, the MPE that we have adopted as benchmark does not capture finer details of the data, such as the initially high levels of investment in both the RIE and IIE regimes, and the relatively high long run stock of public good in IIE. In Section IIIB, we explore these discrepancies by focusing on individual investing behavior.

\section{B. Investing Behavior}

How much do individual agents invest in the public good? Figure 3 shows the time series of the median investment in the public good by treatment. The horizontal axis is the time period and the vertical axis is the investment in the public good. The maximum amount each agent can allocate to investment is the same in each period, and it is given by $W / n$, which is equal to 20 for $n=3$ and 16 for $n=5$. The minimum amount each agent can invest is always zero in the irreversible investment treatment, but it depends on the stock at the beginning of the period in the reversible investment treatment (since each agent can disinvest up to $g / n$ units of the public good). For each period, we use the median level of individual investment from all subjects in a given treatment. Similar results hold if we use the mean or other measures of central tendency.

Figure 3 shows a series of interesting patterns. First, the median individual investment is always higher with irreversible investment than with reversible investment in periods 1-10. Second, the level of investment is decreasing, with median investment converging quickly to values around zero for the reversible investment economies and steadily decreasing toward zero for the irreversible economies.

\footnotetext{
${ }^{24}$ Both reversible and irreversible investment lead to normalized efficiency level significantly different from zero, that is, the efficiency levels predicted by the MPE. As discussed above, while this difference is large for IIE, it is negligible and not economically significant for RIE.

${ }^{25}$ With the parameters of the experiment, the public good stock sustainable with the nonstationary strategies proposed in the proof of Proposition 3 is 520 (versus an efficient level of 576) for $n=3$ and 1,404 (versus an efficient level of 1,600 ) for $n=5$. This steady state is reached in 9 periods (with investment equal to $W=60$ in the first 8 periods) for $n=3$ and in 18 periods (with investment equal to $W=80$ in the first 17 periods) for $n=5$.
} 


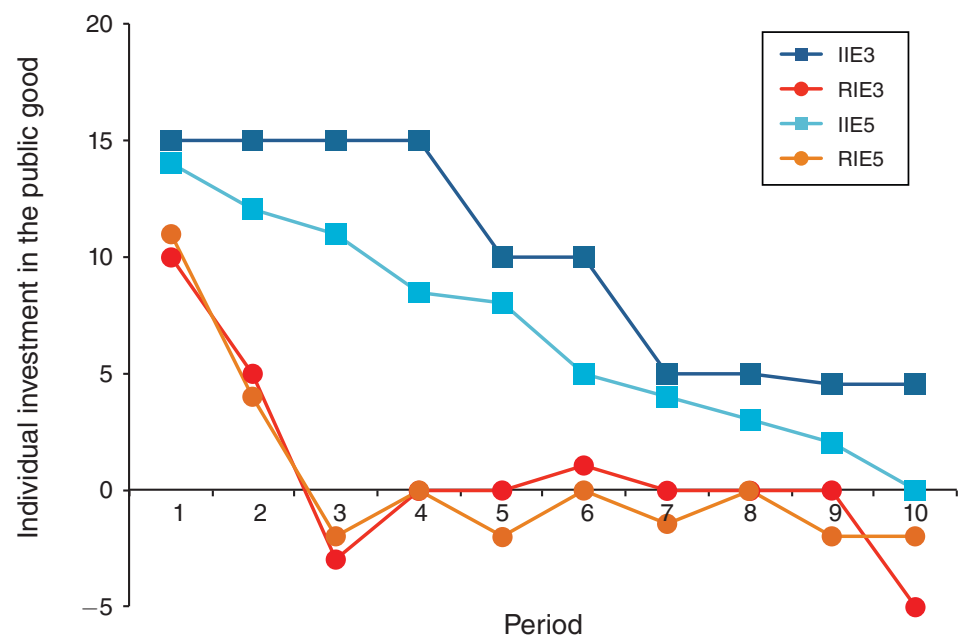

Figure 3. Median Individual Investment

How do these levels of individual investment compare to the theoretical predictions? The median time paths from Figure 3 are qualitatively in line with the predicted time paths: with reversible investment, the theory predicts positive investment only in the first period (when the equilibrium steady state is reached) and zero investment from the second period on; with irreversible investment, the theory predicts positive investment in each period, but at a monotonically decreasing pace (with convergence to the equilibrium steady state only asymptotically). Again, however, there are some differences between the finer details of the theoretical predictions and the data, that mirror the findings in Section IIIA. We observe overinvestment in the early periods: while individual investment is predicted to be less than 4 units in the first period for all treatments, we observe medians between 10 and 15 . In the reversible economies, this overinvestment is corrected in the later periods: the median investment falls sharply to zero or below and a large fraction of individuals disinvests, with higher early overinvestment followed by higher disinvestment. In RIE, negative investment accounts for 28 percent (for $n=3$ ) or 36 percent (for $n=5$ ) of all decisions. The proportion of negative investment decisions increases with the period of play, within a single match.

The game we study is a dynamic game with an evolving state variable. It follows that, to better compare the observed level of investment with the theoretical predictions, we need to take into account the state variable faced by each agent when making an allocation decision, that is the stock of the public good at the beginning of a period. For each subject in each period, we calculate the difference between his observed behavior and the investment predicted by the theory given the public good stock in his group in that period. Figure 4 shows the time series of the median of this difference. This series starts out significantly above zero for all treatments but decreases as more periods of the same match are played, suggesting that subjects' decisions respond to the evolution of the state variable. Notice that this pattern leads to public good outcomes that are in line with MPE steady states for reversible economies, but not for irreversible economies: in the former, subjects can correct the 
Panel A. $n=3$

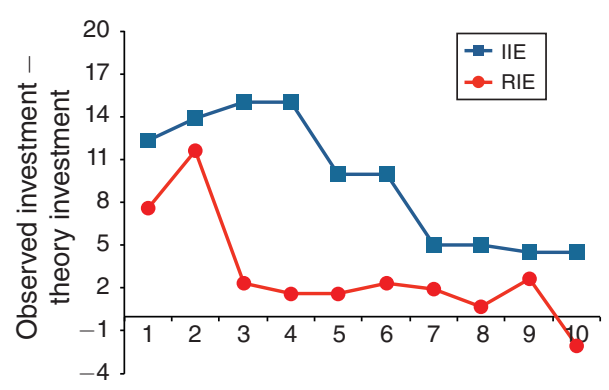

Panel B. $n=5$

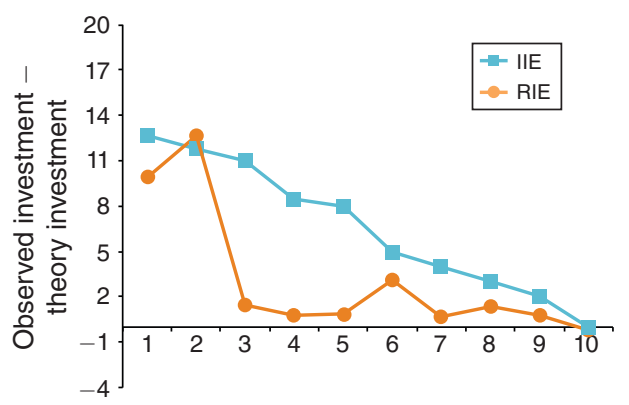

Figure 4. Median Difference with MPE InVEstment

initial overinvestment with negative investment, while in the latter the equilibrium investment for any level above the steady state (64) is bound to be zero and the initial overinvestment persists. We summarize these findings below.

Finding 4: In all treatments, there is overinvestment relative to the equilibrium in the early periods. This is followed by negative investment approaching the theoretical predictions in RIE, while the overinvestment decreases but persists in IIE. In all treatments, groups overshoot the equilibrium in early periods: the difference between the average investment in periods 1-2 and the predicted investment in these same periods is statistically significant at the 1 percent level for all treatments. In $\mathrm{RIE}$, this overshooting is largely corrected in later periods via disinvestment. When investment is reversible, convergence of the public good stock is close to equilibrium, with the difference between the median public good levels and the equilibrium public good levels in the last 4 periods of data measuring around 5 units of the public good with $n=3$ and around 28 units with $n=5$. In IIE, investment remains positive but is monotonically decreasing with periods of play (in the same match). ${ }^{26}$ Given the public good stock by the end of period 2 is already above the predicted steady state level (64), the positive-albeit slower-investment flow in the following periods brings the long-run level of public good to be four (295.5 versus 64 for $n=3$ ) and five times (324 versus 64 for $n=5$ ) larger than predicted. The difference between the average public good stock and the predicted public good stock in periods 6-10 is statistically significant at the 1 percent level.

\section{The Effect of Group Size}

According to the theory, the public good accumulation has a small sensitivity to group size: for both accumulation mechanisms (reversible or irreversible investments), the public good stock is predicted to be larger in smaller groups in every period, but this difference ranges between 1.36 and 2.44 (always less than 5 percent

\footnotetext{
${ }^{26}$ Recall that in the IIE equilibrium, contribution is predicted to be positive in every period, and to monotonically decline to zero.
} 
of the group per period endowment). We discuss below the observed difference in public good accumulation between three-member and five-member groups.

Finding 5: Public good stocks are higher in five member groups than in three member groups. This difference, however, is statistically significant only in half of the periods. For the same accumulation mechanism, the average and median stock of public good is higher with five-member groups than with three-member groups in every single period. However, this difference is statistically significant at conventional levels $(p<0.05)$ only for periods $1,2,5,6,8$, and 9 in RIE; and for periods 1-5 in IIE (see Table 3, columns 3 and 4).

\section{The Effect of Experience}

As the previous sections show, across periods of the same game, subjects' investment behavior gets closer to equilibrium. It is therefore natural to ask whether we observe a similar pattern across matches. Do subjects choose allocations closer to the equilibrium when they are more experienced? Or do they still overinvest in early periods and reduce investment in later periods, even after many matches of the same (multi-period) game?

Finding 6: With reversible investment, public good stocks are not affected by experience. With irreversible investment, public good stocks are higher in later matches. We compare public good stocks in each period in early (1-5) versus late (6-10) matches. Figure 5 shows the median time paths of the public good stock for early and late matches separately, for each treatment. In RIE, as subjects play more matches within the same session the stock of the public good is unaffected in periods $1-10 .{ }^{27}$ In IIE, as subjects play more matches within the same session, the accumulated levels of the public good grow larger: public good stocks are higher in late matches for all periods in both group sizes. With $n=3$, this difference is statistically significant at the 1 percent level in periods 2-7 and 9, at the 5 percent level in periods 8 . With $n=5$, this difference is statistically significant at the 1 percent level in period 1 and 3 , at the 5 percent level in period 2 .

\section{Explaining Differences with MPE}

The aggregate outcomes presented in the previous section reflect the main qualitative features from the MPE: both in RIE and IIE the accumulation path is inefficiently slow, the public good is under-provided, and IIE induces significantly higher public good contributions than RIE. On the other hand, the MPE does not fully capture the complexity of individual behavior: the levels of public good stock are far from efficient but also far from the predictions of the Markov perfect equilibrium in the IIE environment; under RIE, public good levels exhibited a time path of early

\footnotetext{
${ }^{27}$ The only significant difference is in period 3 for $n=3$, where the difference is significant at the 1 percent level. Notice that, for RIE, we are testing simultaneously 17 hypotheses and the probability of observing 1 significant result just because of chance is 58 percent.
} 


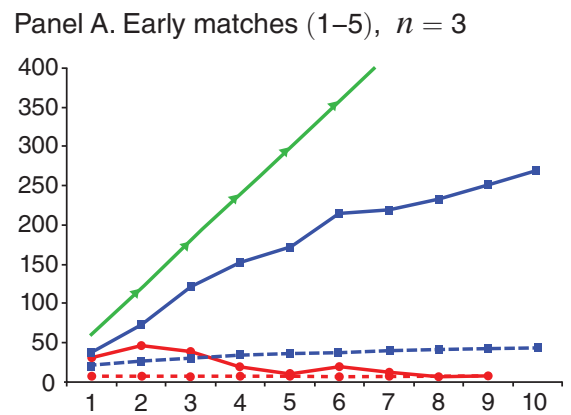

Panel B. Early matches (1-5), $n=5$

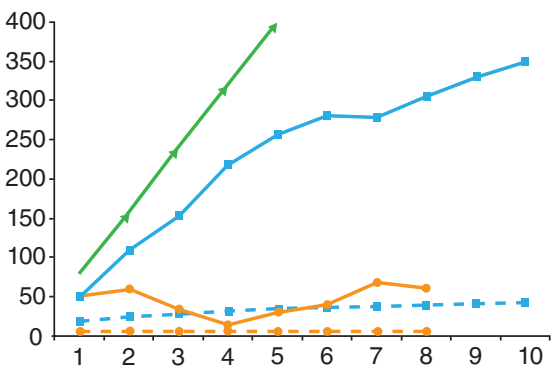

- - - IIE theory $\rightarrow$ IIE obs. mdn. $\longrightarrow$ Planner
.- RIE theory $\rightarrow$ RIE obs. mdn.

Panel C. Late matches (6-10), $n=3$
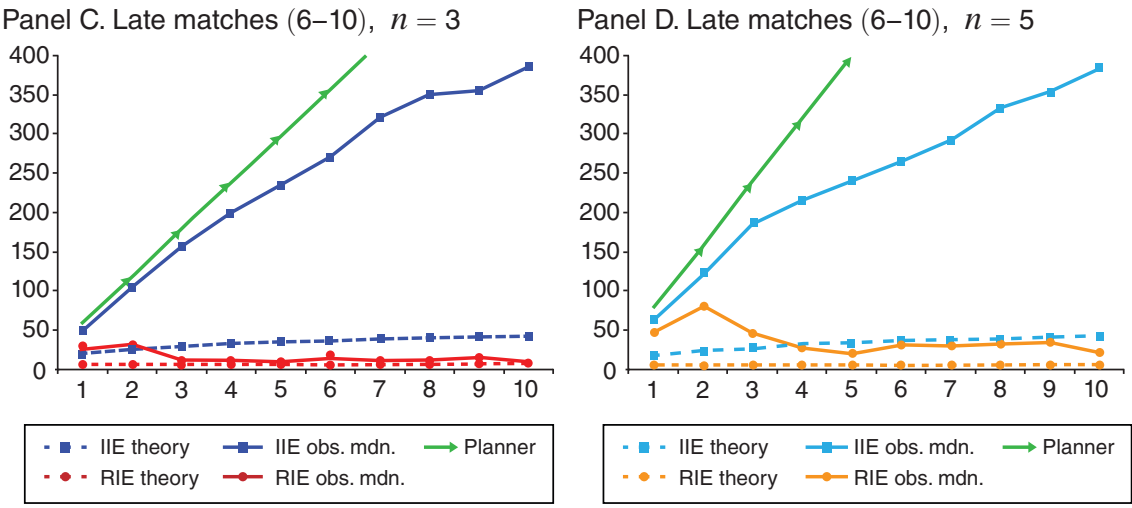

Figure 5. Median Time Paths of the Stock of $g$, the Effect of Experience

overproduction followed by negative investment. This is suggestive of some amount of cooperation that may be accountable in part by a failure of the Markovian assumption and by nonstationary strategic behavior involving punishments and rewards.

In this section, we investigate this possibility with two complementary methodologies. First, we look more closely at the individual investing behavior in the dynamic games: we test whether current individual investment decisions are correlated to past investments by other group members. Second, we propose a novel experimental test of the Markovian assumption: we design a one-period experiment where subjects' payoffs from the public good are given by the equilibrium value function of the unique concave Markov perfect equilibrium of the game with reversibility. If subjects in the fully dynamic game condition their strategies only on the public good stock, the individual incentives to contribute in the public good are exactly the same and we should observe no difference in behavior in this new treatment.

\section{A. History-Dependent Behavior}

The tools by which players in this voluntary contribution game can reward or punish the other agents are limited. This is because punishments cannot be "targeted": an individual agent can only punish/reward other agents collectively by investing less/more in the public good in future periods. With this in mind, we 
Table 4-Tobit Estimates for Individual InVestment Decisions

\begin{tabular}{lcccc}
\hline \hline & $(1)$ & $(2)$ & $(3)$ & $(4)$ \\
\hline Public good stock & 0.009 & -0.043 & -0.008 & -0.010 \\
& $(0.028)$ & $(0.016)$ & $(0.003)$ & $(0.001)$ \\
Mean(Investment $)_{t-1}$ & 0.228 & 0.286 & 1.286 & 1.358 \\
& $(0.050)$ & $(0.060)$ & $(0.058)$ & $(0.095)$ \\
SD(Investment) ${ }_{t-1}$ & -0.729 & -0.946 & -0.281 & -0.278 \\
& $(0.105)$ & $(0.107)$ & $(0.080)$ & $(0.130)$ \\
Constant & -0.316 & 13.026 & 1.518 & 4.226 \\
& $(0.988)$ & $(2.876)$ & $(1.132)$ & $(1.375)$ \\
Treatment & & & & IIE3 \\
Subject FE & RIE3 & RIE5 & Yes & Yes \\
Pseudo $R^{2}$ & Yes & Yes & 0.1910 & 0.2182 \\
Observations & 0.0456 & 0.0574 & & 1,440 \\
\hline
\end{tabular}

Note: Standard errors clustered by groups are in parentheses.

regress current individual investment decisions on last period's average investment in their group, controlling for the level of public good. A positive coefficient would be consistent with some sort of nonstationary behavior, such as collective punishments and rewards. We also include last period's standard deviation of investment decisions in their group, as a high variance will indicate the presence of shirkers in their group, which could trigger (untargeted) punishments. A negative coefficient would be consistent with untargeted punishment of individual shirking behavior. Table 4 shows the results for each treatment. An observation is a single subject's allocation decision in a single period. We include subject fixed effects to control for individual heterogeneity and cluster standard errors by group to take into account possible correlations among decisions taken by the same group.

Finding 7: Investment decisions depend on the contributions of others. The results for the four treatments are similar. The public good stock, as well as both "punishment" variables have the predicted sign. With the exception of RIE with $n=3$, the coefficient of the current stock of the public good is negative and significant. The average lagged investment is positive and highly significant in all treatments. The magnitude of its coefficient is around four times larger in IIE than in RIE. The lagged standard deviation coefficient is negative and significant in all treatments. The magnitude of its coefficient is around two (for $n=3$ ) and three (for $n=5$ ) times larger in RIE than in IIE. This seems to suggest nonstationary behavior that may be consistent with strategic attempts to maintain higher-than-equilibrium investment levels. To the extent that these attempts may have increased investment levels, the magnitude of such an increase is rather small in RIE but substantial in IIE, which probably accounts for some part of the large difference between IIE and RIE.

\section{B. Direct Test of Markovian Behavior}

To what extent are the models we use adequate to study this problem? What equilibrium concepts should be used? The latter question is particularly important 
in these dynamic games since, depending on the equilibrium concept, we can have very different predictions for the same model. While it is difficult to identify the equilibrium adopted by players, the analysis of public good outcomes and investing behavior provides some insights. As discussed above, we observe a consistent pattern of behavior across groups, despite the fact that we have multiplicity of potential equilibria; with the exception of initial overinvestment, the investing behavior is tied to the evolution of the stock as qualitatively predicted by the theory; and, at least for RIE, the long term public good outcomes are close to the equilibrium steady states.

To further pursue this question, we construct a more direct test of the Markovian restriction, that is, of the assumption that players are forward-looking and condition their strategy only on the stock of the public good at the beginning of the period, irrespective of the histories. In particular, we conduct a one-period version of the reversible investment game, where the payoffs from the public good stock are complemented by the equilibrium value functions of the unique concave Markov perfect equilibrium of the game. In each one-period game, agent $j$ receives the following payoff:

$$
U^{j}\left(x^{j}, y\right)=x^{j}+\alpha \sqrt{y}+\delta v_{R}(y)
$$

where $x^{j}$ is the private consumption of agent $j, y$ is the end-of-period public good stock, and $\delta v_{R}(y)$ is the discounted equilibrium value function from the dynamic game with reversible investment.

In each experimental session, subjects play for 40 matches. Contrary to the dynamic game, the length of each match is known and equal to one period. At the end of each one-period match, subjects are reshuffled into new groups and the public good stock starts out at a (potentially different) exogenous level. We use eight different $g_{0}$, to elicit an investment strategy (as a function of the state variable) comparable to the one observed in the fully dynamic game. Table 5 below summarizes the experimental design.

In each experimental session, each of the 8 values of $g_{0}$ is used in 5 different matches, in random order, for a total of 40 matches. The range [0-35] covers around 75 percent of observations in the dynamic game with 3-member groups and around 55 percent of observations in the dynamic game with 5-member groups. In the one-period reduced form treatments, the unique equilibrium of the game prescribes the same investment level predicted for the fully dynamic game under the Markovian assumption that subjects condition their strategies only on the public good stock. While there is no other equilibrium in this one-period game, in the fully dynamic game there is a plethora of different subgame perfect equilibria that can sustain a higher level of investment with nonstationary strategies. Therefore, if we observe similar behavior in the two treatments, this provides some additional evidence for Markovian strategies in the fully dynamic game, as opposed to strategies that depend on history in a non-Markov way. On the other hand, any differences in behavior provide indirect evidence for the presence of non-Markov strategies that can arise in dynamic games. 
Table 5-Experimental Design, One-Period Reduced-Form Treatments

\begin{tabular}{ccccc}
\hline \hline$n$ & $W$ & Groups & Subjects & $g_{0}$ \\
\hline 3 & 60 & 80 & 24 & $0,5,10,15,20,25,30,35$ \\
5 & 80 & 60 & 30 & $0,5,10,15,20,25,30,35$ \\
\hline
\end{tabular}

Panel A. $n=3$

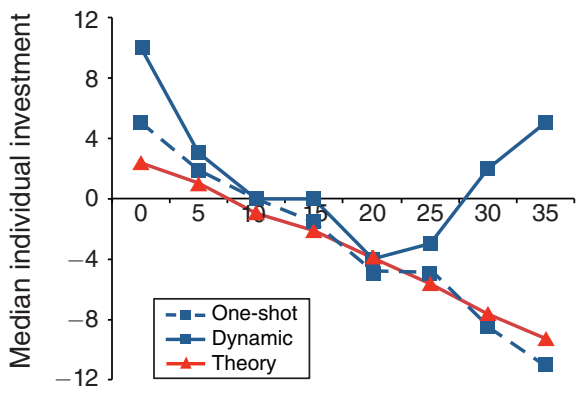

Beginning-of-period public good stock
Panel B. $n=5$

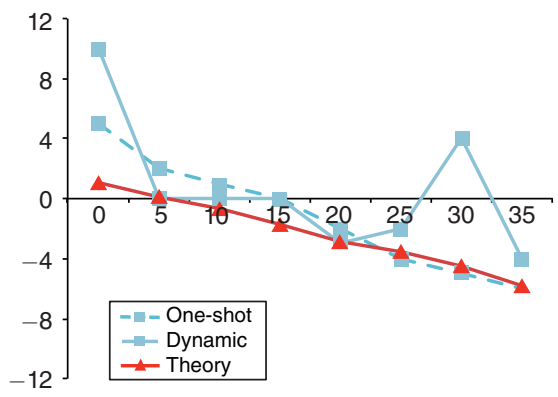

Beginning-of-period public good stock

Figure 6. Median Investment as a Function of Beginning-OF-Period Stocks

Figure 6 illustrates the median individual investment as a function of the initial stock for the one-period reduced-form games described above and for the fully dynamic games. 28

Finding 8: For intermediate values of public good stock, the dynamic and static experiments produce similar results; for extreme values of the stock, the dynamic experiment produces a higher level of contributions. For 3-member groups, investment is significantly higher at the 5 percent level in the dynamic treatment for initial stocks of $0,25,30$, and 35 , and statistically indistinguishable for the remaining initial stocks. For 5-member groups, investment is significantly higher at the 5 percent level in the dynamic treatment for an initial stock of 30, and statistically indistinguishable for the remaining initial stocks. ${ }^{29}$

While there are some significant differences, these differences are small in magnitude (with the exception of initial stocks greater than 25 for $n=3$ ). This provides a mixed picture, but is suggestive that investment is somewhat higher in the fully dynamic game than in the reduced form game, perhaps as a consequence of nonstationary strategies. Of course, focusing on medians masks a lot of

\footnotetext{
${ }^{28}$ Since the beginning-of-period stock in the dynamic games is endogenous and does not necessarily match the values used in the one-period games, for these games we use the median investment levels for all periods-groups that started with a public good stock in a six experimental units interval around the starting size used in the one-period games. For example, the median investment corresponding to a beginning-of-period stock of 20 is computed as the median investment from all periods-groups starting at a stock between 17 and 23. This allows us to have a comparable number of observations between one-period and dynamic games.

2 Table 6 reports the $p$-values of these tests. If we use a 10 percent significance level, for both group sizes, investment is significantly higher in the dynamic treatment for initial stocks of $0,25,30$, and 35 , and statistically indistinguishable for the remaining initial stocks.
} 
heterogeneity across groups. In fact, the median investment in the one-shot treatment is in the interquartile range of the investments observed in the dynamic game for all initial stocks in both treatments, with the lone exception of an initial stock of 35 for the 3-member groups. Regarding the high investment in the dynamic treatment for 3-member groups and stocks greater than 25, this is due to a few groups who invested significantly more heavily than predicted by the Markov perfect equilibrium; furthermore, this only happened rarely because most of the observations from the dynamic treatment (where the initial public good stock is endogenous) have a beginning-of-period stock of 25 or less. ${ }^{30}$

\section{Static versus Dynamic: What Have We Learned?}

While this is the first experimental study of the dynamic accumulation process of a durable public good, a vast experimental literature has addressed the provision of public goods in static environments. This begs the following questions: How do the results from our dynamic public good experiments compare to the results from the repeated static public good games? What new insights can we learn breaking out from the static framework?

Comparing directly our dynamic game with the static framework used by the previous experimental literature is generally a difficult task, for a number of reasons. Even when static public good games are repeated a fixed number of times, the strategic environment is the same in every period, and there is a unique equilibrium prediction, that does not change over time. The most common example is the voluntary contribution game with linear payoffs, in which the individually optimal investment level is zero, while the socially optimal one is the whole budget. The equilibrium is in dominant strategies, and there are a finite number of periods, so contributions in past and future periods do not matter for equilibrium behavior, and agents' expectations about other agents' current or future contributions are irrelevant from the standpoint of subgame perfect equilibrium. ${ }^{31}$ The game we study, on the other hand, is not only an infinite horizon game, but a stochastic game with an evolving state variable, and a strategic environment that changes in every period (as the durable public good is accumulated over time). Our theory makes predictions that are path dependent and change over time (equilibrium investments are sometimes positive, sometimes negative, and sometimes zero, depending on the current stock of public good), and, while we restrict attention to the unique concave MPE, the infinite horizon of the game gives rise to a plethora of nonstationary equilibria that have much different properties. In this dynamic setting, not contributing is socially optimal in some continuation games (when the stock of the public good has reached the optimal level). More importantly, at any point in time, even in a Markov equilibrium,

\footnotetext{
${ }^{30}$ Since the beginning-of-period stock in the dynamic treatment is endogenous we have a reduced number of observations for these high values: we use only 18 groups to compute the median investment for a starting stock of 35. The remaining 92 groups never accumulated these levels of public good. The beginning-of-period stock is smaller than 25 in 66 percent of observations (regardless of period number). The average beginning-of-period public good stock in periods $8-10$ (that is, the long run level of public good) is 15.9 .

${ }^{31}$ This applies as well to some of the dynamic games based on the linear voluntary contributions model, including the variation with no completion benefit in Duffy, Ochs, and Vesterlund (2007).
} 
Table 6-Average Investment as a Function of Beginning-OF-Period Stocks

\begin{tabular}{|c|c|c|c|c|c|c|}
\hline \multirow[b]{2}{*}{$g_{0}$} & \multicolumn{3}{|c|}{ RIE 3} & \multicolumn{3}{|c|}{ RIE 5} \\
\hline & Dynamic & One-shot & $p$-value & Dynamic & One-shot & $p$-value \\
\hline 0 & $9.54(342)$ & $6.47(120)$ & 0.016 & $9.48(300)$ & $7.22(150)$ & 0.083 \\
\hline 5 & $2.98(63)$ & 4.07 (120) & 0.935 & $2.31(55)$ & $4.88(150)$ & 0.302 \\
\hline 10 & $0.70(93)$ & $2.08(120)$ & 0.890 & $0.51(55)$ & $3.73(150)$ & 0.104 \\
\hline 15 & $0.45(96)$ & $0.65(120)$ & 0.200 & $1.90(80)$ & $2.75(150)$ & 0.777 \\
\hline 20 & $-1.56(90)$ & $-2.09(120)$ & 0.217 & $-1.67(45)$ & $0.25(150)$ & 0.533 \\
\hline 25 & $-0.91(78)$ & $-3.73(120)$ & 0.026 & $0.14(65)$ & $-0.13(150)$ & 0.067 \\
\hline 30 & $0.53(93)$ & $-5.53(120)$ & 0.000 & $1.20(40)$ & $-1.43(150)$ & 0.034 \\
\hline 35 & $4.21(63)$ & $-7.51(120)$ & 0.000 & $-0.70(50)$ & $-2.29(150)$ & 0.055 \\
\hline
\end{tabular}

Notes: The number of observations is in parentheses. $p$-values refer to Wilcoxon-Mann-Whitney tests on the equality of distributions with standard errors clustered by individual.

the individually optimal decision depends on past contributions through their effect on the current state, as well as on the expectations on current and future contributions of other agents. Moreover, a fundamental question of our paper, the impact of investment reversibility on the dynamic free rider problem, cannot be studied in a static framework where the public good starts out at zero in every period (and, thus, contributions can only be nonnegative).

In spite of these clear difficulties in comparing the two frameworks, we can still draw some connections between the behavior observed in repeated static public good games and behavior in our dynamic durable public good experiments. In the reminder of this section we discuss a few of the most significant similarities and differences.

Overinvestment, Efficiency, and Irreversibility.-The first has to do with the general issue of whether contributions tend to be above, below, or approximately equal to the theoretically predicted levels. In static environments, with few exceptions, actual contributions are generally above the equilibrium levels suggesting that equilibrium theory tends to overpredict the amount of free riding. ${ }^{32}$ Still, contributions not only fail to reach efficient levels (as Ledyard 1995 reports in his survey), but are generally very much below. Average contributions in initial plays of the game typically fall in a range between 40 percent and 60 percent of the optimal level, with a systematic decline to very low levels with repetition (between 10 percent and 20 percent of the optimal level after 10 periods of play). Similarly, in our dynamic environment, there is significant over-contribution with respect to the predictions of the unique MPE in the early periods of play, while the public good stock is beginning to accumulate, but this over-contribution mostly disappears in later periods (especially with reversible investment, where investments become negative and the long run stock of the public good converges to very close to the MPE steady state). These declines over time in both the reversible and irreversible cases lead to significantly inefficient long-run public good levels (see Figure 2, and Findings 1 and 2). How

\footnotetext{
${ }^{32}$ There are a few exceptions. Laury and Holt (2008) investigate nonlinear, static public good technologies with interior equilibrium contributions. Palfrey and Rosenthal (1991) explore threshold public goods with binary contribution decisions. For some of the treatments in these two papers, contributions are less than equilibrium predictions.
} 
serious is the inefficiency with a durable public good, as compared to one-shot public goods problems? Interestingly, the answer depends critically on whether contributions are reversible. With reversibility, the median public good stock converges to approximately 2 percent of the efficient steady state. In contrast, with irreversibility, the median period 10 stock of public good is approximately 50 percent of the efficient level. Thus, with reversible investments we see efficiency levels that are worse than is typically observed in static voluntary contribution games, but the opposite is the case with irreversible investment. This very strong effect of irreversibility, with important consequences in real world applications of the theory, is not observable in static laboratory environments or in repeated games without a state variable.

Investment Pattern and Dynamics.-Second, there are some similarities in terms of the investment pattern we observe over time: as in the static literature, in our dynamic experiments, there is a tendency for initial overinvestment in the early periods, followed by investment levels approaching the theoretical predictions (see Figures 4 and 5). Moreover, a similar pattern is observed when subjects are re-matched into new groups and the public good stock starts out at zero, a phenomenon similar to the "re-start effect" from the static literature (see Andreoni and Croson 2008 for a survey).

Contrary to much of the static literature-where the predictions are no contributions in every period - the time paths we observe in the dynamic games qualitatively follow the same pattern as the predicted time paths, especially in later periods. With reversible investment, the theory predicts positive investment only in the first period and zero investment from the second period on. With irreversible investment, the theory predicts positive investment in each period, but at a monotonically decreasing pace. These general patterns are found in our data. Also, the convergence to the equilibrium predictions follows a different pattern from static experiments and the equilibrium predictions themselves are path dependent and endogenous: in the treatment with reversibility, we observe significant levels of negative investment, with subjects reacting to above-equilibrium accumulation levels and the stock of public good gradually declining in the direction of the equilibrium steady state.

Heterogeneity in Behavior.-Finally, another finding of the experimental literature on static public good games is the existence of distinct types of behavior. This was first considered by Isaac, Walker, and Thomas (1984), who classify each investment decision as being "Strong Free-Riding," "Weak Free-Riding," or "Lindahl/ Altruistic" depending on whether the investment is less than 33 percent, between 33 percent and 66 percent, and more than 66 percent of the individual budget, respectively. ${ }^{33}$ According to this classification, they report 44 percent of investment decisions in their experiment are Strong Free-Riding, 27 percent are Weak Free-Riding, and 29 percent are Lindahl/Altruistic. We applied a similar analysis to our data (adjusting for the fact that the individual budget in the reversible investment treatments is state-dependent) and we found a rather similar distribution of decision

\footnotetext{
${ }^{33}$ They do not classify individuals into behavioral types based on their behavior in the ten periods of play.
} 
types (pooling all treatments together): 42 percent of investment decisions in our experiments are Strong Free-Riding, 18 percent are decisions are Weak Free-Riding, and 41 percent are Lindahl/Altruistic.

A different approach traces these aggregate patterns of contribution behavior to heterogeneity at the individual level. For example, there is some evidence from static public good experiments that some individuals behave as "conditional cooperators," whose contribution to the public good is positively correlated with their beliefs about the contributions made by their group members (Keser and Van Winden 2000; Fischbacher, Gächter, and Fehr 2001; Burlando and Guala 2005; Fischbacher and Gächter 2010). ${ }^{34}$ While subjects in our experiment are not explicitly asked to make decisions contingent on the other group members' contributions, over the course of the experiment they experience a wide range of (endogenous) past group decisions and we can use this data to measure the extent to which individual contributions respond positively to other group members' past contributions. There is also evidence for the existence of other behavioral types who are either altruistic or completely selfish.

To identify these different behavioral types, we follow Fischbacher and Gächter (2010) and, for each subject, we estimate a Tobit regression of the deviation from predicted investment (notice that, in the static experiment, this is simply equal to the investment level) on the average investment of the other group members in the previous period (with a constant). We then classify each subject as a conditional cooperator if the slope is positive and significantly different than zero at the 1 percent level; as an "unconditional cooperator" if the slope is not significantly different than zero and his average investment deviation is in the top third of the distribution; as "free rider" if the slope is not significantly different than zero and his average investment deviation is in the bottom third of the distribution. The results are reported in Table 7. Overall, we measure 53 percent conditional cooperators, 14 percent unconditional cooperators, 15 percent free riders, and 18 percent unclassified. This distribution of behavioral types is roughly in line with the static literature, although there is considerable variation. ${ }^{35}$ The fact that we measure a large percentage of conditional cooperators offers some additional evidence for non-Markovian strategies. A second result from Table 7 is the difference between reversible and irreversible investment treatments: conditional cooperators account for 79 percent of subjects in IIE but only for 27 percent of subjects in RIE. This is an interesting feature of the data which might contribute to the large difference in observed public good accumulation between the two treatments and which we do not have a good explanation for.

\footnotetext{
${ }^{34}$ There are different possible interpretations for these behavioral types, such as imitation, conformity, reciprocity, repeated game strategies, etc.

${ }^{35}$ The fraction of conditional cooperators in those other studies is usually around 50-60 percent, but ranges from 35 percent (Burlando and Guala 2005) to 80 percent (Keser and Van Winden 2000).
} 
Table 7-Classification of Subjects' Strategies

\begin{tabular}{lllllr}
\hline \hline & IIE3 & IIE5 & RIE3 & RIE5 & Overall \\
\hline Unconditional cooperator & $0.03(1)$ & $0.13(4)$ & $0.15(5)$ & $0.27(8)$ & $0.14(18)$ \\
Conditional cooperator & $0.81(29)$ & $0.77(23)$ & $0.24(8)$ & $0.30(9)$ & $0.54(69)$ \\
Free rider & $0.11(4)$ & $0.07(2)$ & $0.27(9)$ & $0.13(4)$ & $0.15(19)$ \\
Other & $0.06(2)$ & $0.03(1)$ & $0.33(11)$ & $0.30(9)$ & $0.18(23)$ \\
\hline
\end{tabular}

Note: The number of subjects is in parentheses.

\section{Conclusions}

This paper investigated the dynamic accumulation process of a durable public good in a voluntary contribution setup. Despite the fact that most, if not all, public goods are durable and have an important dynamic component, very little is known on this subject, both from a theoretical and empirical point of view. We attempt to provide some initial empirical findings about voluntary contribution behavior with durable public goods.

We have considered two possible cases: economies with reversible investments (RIE), in which in every period individual investments can either be positive or negative; and economies with irreversible investments (IIE), in which the public good cannot be reduced. Reversibility is an important feature of many public goods problems (for example, common pool problems), which is completely missed by static analysis. We also have a secondary treatment dimension: we compare three-member and five-member groups. For all treatments, applying results from Battaglini, Nunnari, and Palfrey (2014) to our laboratory environment, we have characterized the steady states and the accumulation paths that can be supported by the optimal solution and by the unique symmetric concave Markov equilibrium.

We highlight three main results. First, the dynamic free riding problem exists and it is severe, with the long run public good stock levels falling far short of efficiency in all treatments. The additional free riding component that emerges in this dynamic game is most obviously seen in reversible investment economies. With reversibility, the dynamic dimension exacerbates the free rider problem present in static public good provision: if an agent contributes above the equilibrium levels, not only does this reduce the future contributions by all agents, but it triggers an extreme form of dynamic crowding out: negative investment by other agents that transform part of the public good stock in private consumption. In these RIE treatments, the median public good stock converges to approximately 2 percent of the efficient steady states, versus long-run contributions between 10 percent and 20 percent of the optimal level in finitely repeated static public good games.

On the other hand, in line with the comparative static predictions, irreversible investment leads to significantly higher public good production than reversible investment. The irreversibility constraint dampens the dynamic free rider problem, by creating a commitment device and reducing the crowding-out effect of contributions. Notice that this has nothing to do with history dependent trigger strategies made possible by the infinite horizon: a similar dynamic would arise in a model with a finite horizon (but losing stationarity of equilibrium strategies). In the IIE 
environment where the public good cannot be converted back to consumption, the median period 10 stock of the public good is approximately 50 percent of the efficient level.

Second, we have shown that, in both the RIE and IIE environments, there is overinvestment in the early periods, compared to the equilibrium investment levels. In RIE, this is followed by a significant reversal, with the stock of public good gradually declining in the direction of the equilibrium steady state. In IIE, where disinvestment is not feasible, investment steadily decreases but the initial overinvestment cannot be reversed and the long-run level of the public good remains significantly above the equilibrium steady state.

Third, we have proposed a novel experimental methodology to test the assumption that subjects' strategies in this complex infinite-horizon game depend only on the state variable, that is, the accumulated level of the public good.

This is the first experimental study of the dynamic accumulation process of a durable public good. Our design was intentionally very simple and used a limited set of treatments. As a consequence, there are many possible directions for the next steps in this research. The theory has interesting comparative static predictions about the effect of other parameters that we have not explored in this work, such as the discount factor, the depreciation level, preferences, and endowments. For example, a higher discount factor increases both the optimal steady state and the equilibrium steady state of the durable public good for all values of $n$ and for both reversible and irreversible economies. For similar reasons, positive depreciation in the public good technology leads to a decrease in the steady state of the Markov equilibrium studied here. Among these extensions, it would be particularly interesting to run experiments that allow a closer comparison with the results from the static literature. This can be done in a number of different ways: for example, experiments with a finite and known horizon of one period (that is, $\delta=0$ ), or experiments with full depreciation of the stock at the end of each period and an infinite horizon (that is, $\delta>0$ ).

Moreover, our model and experimental design does not consider different rules for negative investment (for example, allowing subjects to disinvest unilaterally up to the whole stock and adopting a rationing rule to keep a nonnegative level of public good), or the effect of a completion benefit at a specified accumulation threshold. We have also limited the analysis to voluntary contribution mechanisms that turn out to be highly inefficient, both in theory and in practice. Battaglini, Nunnari, and Palfrey (2012) study how centralized mechanisms fare in providing durable public goods and show that efficiency increases with the majority rule required to approve an allocation decision. An interesting direction to pursue from here would be to consider different decentralized mechanisms and explore which ones are more efficient for the provision of durable public goods. 


\section{Appendix A: Proofs of Propositions}

\section{A. Proof of Proposition 1}

The fact that a strictly concave equilibrium has the property stated in the proposition follows from the discussion in the text. Here we prove existence and uniqueness.

Existence.-First note that when we use the functional form $u(g)=\alpha \sqrt{g}$, we have $u^{\prime}(g)=\frac{\alpha}{2 \sqrt{g}}$ and $\left[u^{\prime}\right]^{-1}(x)=\left(\frac{\alpha}{2 x}\right)^{2}$. Battaglini, Nunnari, and Palfrey (2014) show that there is a weakly concave Markov equilibrium with steady state equal to $y_{R}^{*}$ for any $y_{R}^{*} \in\left[\left(\frac{\alpha n}{2(n-\delta)}\right)^{2},\left(\frac{\alpha}{2(1-\delta)}\right)^{2}\right]$. To prove that the equilibrium corresponding to the steady state $y_{R}^{*}=\left(\frac{\alpha n}{2(n-\delta)}\right)^{2}$ is strictly concave, we provide an alternative (self contained) existence proof.

Let $y_{R}^{*}=\left(\frac{\alpha n}{2(n-\delta)}\right)^{2}$ and $g_{R}^{1}=\max \left\{0, y_{R}^{*}-W\right\}$. For any $g>g_{R}^{1}$ define a value function $v_{R}^{1}(g)=\frac{W-\left(y_{R}^{*}-g\right)}{n}+\frac{1}{1-\delta} \alpha \sqrt{y_{R}^{*}}+\delta \frac{W}{n}$. Note that this function is continuous, nondecreasing, weakly concave, and differentiable with respect to $g$, with derivative $\left[v_{R}^{1}\right]^{\prime}(g)=\frac{1}{n}$. From strict concavity of $u(g)$ it follows that, for any $g>g_{R}^{1}$, the objective function in (2) is strictly concave. Let $g_{R}^{2}=\max \left\{0, g_{R}^{1}-W\right\}$, and define

$$
v_{R}^{2}(g)=\left\{\begin{array}{cc}
v_{R}^{1}(g) & g \geq g_{R}^{1} \\
\alpha \sqrt{g+W}+\delta v_{R}^{1}(g+W) & g \in\left[g_{R}^{2}, g_{R}^{1}\right)
\end{array} .\right.
$$

Note that $v_{R}^{2}(g)$ is continuous and differentiable in $g \geq g_{R}^{2}$, except at most at $g_{R}^{1}$. To see that the objective function in (2) is strictly concave in this interval, note that it is strictly concave for $g \geq g_{R}^{1}$. Moreover, for any $g \in\left[g_{R}^{2}, g_{R}^{1}\right)$ and $g^{\prime} \geq g_{R}^{1}$ we have

$$
\begin{aligned}
{\left[v_{R}^{2}\right]^{\prime}(g) } & =\frac{\alpha}{2 \sqrt{g+W}}+\delta\left[v_{R}^{1}\right]^{\prime}(g+W) \\
& >\frac{\alpha}{2 \sqrt{y_{R}^{*}}}+\delta\left[v_{R}^{1}\right]^{\prime}\left(y_{R}^{*}\right)=1>\frac{1}{n}=\left[v_{R}^{1}\right]^{\prime}(g) .
\end{aligned}
$$

The first inequality derives from $y_{R}^{*}>g+W$ (which is true, by definition of $g_{R}^{1}$ and $g_{R}^{2}$, for all $\left.g \in\left[g_{R}^{2}, g_{R}^{1}\right)\right)$, strict concavity of $u(g)$ and weak concavity of $v_{R}^{1}(g)$. It follows that $u(g)+\delta v_{R}^{2}(g)$ is strictly concave in $g \geq g_{R}^{2}$. Assume that for all $g \geq g_{R}^{n}$, with $g_{R}^{n} \geq 0$ and either $g_{R}^{n}<g_{R}^{2}$ or $g_{R}^{n}=0$, we have defined a value 
function $v_{R}^{n}(g)$ that is concave and continuous, and that is differentiable in $g>g_{R}^{1}$. Define $g_{R}^{n+1}=\max \left\{0, g_{R}^{n}-W\right\}$, and

$$
v_{R}^{n+1}(g)=\left\{\begin{array}{cc}
v_{R}^{n}(g) & g \geq g_{R}^{n} \\
\alpha \sqrt{g+W}+\delta v_{R}^{n}(g+W) & g \in\left[g_{R}^{n+1}, g_{R}^{n}\right)
\end{array} .\right.
$$

Using the same steps as above, we can easily show that this function is weakly concave, continuous in $g \geq g_{R}^{n+1}$, and differentiable for $g>g_{R}^{1}$. Moreover, either $g_{R}^{n+1}=0$ or $g_{R}^{n+1}<g_{R}^{n}$. We can therefore define inductively a value function $v_{R}(g)$ for any $g \geq 0$ that is continuous, weakly concave, and that is differentiable at least for $g>g_{R}^{1}$, and so, in particular, at $y_{R}^{*}$. This value function will give rise to an objective function in (2), which is strictly concave. Define now the following strategies:

$$
y_{R}(g)=\min \left\{W+g, y_{R}^{*}\right\}, \quad \text { and } \quad x_{A}(g)=\left[W+g-y_{R}(g)\right] / n .
$$

We will argue that $v_{R}(g), y_{R}(g), x_{A}(g)$ is an equilibrium. To see this, note that by construction, if the agent uses strategies $y_{R}(g), x_{A}(g)$, then $v_{R}(g)$ describes the expected continuation value function of an agent. To see that $y_{R}(g), x_{A}(g)$ are optimal given $v_{R}(g)$, note that for $g \geq g_{R}^{1},\left\{y_{R}^{*}, \frac{W+g-y_{R}^{*}}{n}\right\}$ maximizes (2) when all the constraints except the second are considered; and for $g \geq g_{R}^{1}, W+g>y_{R}^{*}$, so the second constraint is satisfied as well. For $g<g_{R}^{1}$, we must have $y_{R}(g)=W+g, x_{A}(g)=0$. We conclude that $y_{R}(g), x_{A}(g)$ is an optimal reaction function given $v_{R}(g)$.

Uniqueness. - Consider a strictly concave equilibrium with value function $v_{R}(g)$. Because $u(g)+\delta v_{R}(g)-g$ is strictly concave, there is a unique maximum $y_{R}^{*}$ of the objective function of (2). It follows that we must have $y_{R}(g)=\min \left\{W+g, y_{R}^{*}\right\}$, implying that $y_{R}(g)=y_{R}^{*}$ for any $g \geq y_{R}^{*}-W$ and $y_{R}\left(y_{R}^{*}\right)=y_{R}^{*}$. It is straightforward to show that the derivative of the value function in $g \geq y_{R}^{*}-W$ exists and it is equal to $v_{R}^{\prime}(g)=1 / n$. Using the first-order condition that defines $y_{R}^{*}$, we must have $\frac{\alpha}{2 \sqrt{y_{R}^{*}}}+\delta v_{R}^{\prime}\left(y_{R}^{*}\right)=1$. This implies that in any strictly concave Markov equilibrium we must have a steady state $y_{R}^{*}=\left(\frac{\alpha n}{2(n-\delta)}\right)^{2}$.

\section{B. Proof of Proposition 2}

Since the equilibrium is weakly concave, we must have that $v_{I R}(g)$ admits a right and left derivative at any point $g$. Let us call $y_{I R}^{+}\left(y_{I R}^{*}\right)$ and $y_{I R}^{-}\left(y_{I R}^{*}\right)$ the, respectively, right and left derivatives. Since at $y_{R}^{*}$ we must have $y_{I R}\left(y_{I R}^{*}\right)=y_{I R}^{*}$, it is easy to see that $y_{I R}^{+}\left(y_{I R}^{*}\right)=1$, since $y_{I R}\left(y_{I R}^{*}+\Delta\right)=y_{I R}^{*}+\Delta$.

Consider now the left derivative. In a left neighborhood of $y_{I R}^{*}$, we must have $y_{I R}(g) \in(0, W+g)$, so $x_{I R}(g)>0$ and

$$
y_{I R}(g) \in \underset{y}{\arg \max }\left\{\alpha \sqrt{y}+\delta v_{I R}(y)-y\right\} .
$$


We can write

$$
\begin{aligned}
v_{I R}(g) & =\frac{W+g-y_{I R}(g)}{n}+\alpha \sqrt{y_{I R}(g)}+\delta v_{I R}(g)\left(y_{I R}(g)\right) \\
& =\alpha \sqrt{y_{I R}(g)}+\delta v_{I R}(g)\left(y_{I R}(g)\right)-y_{I R}(g)+\frac{W+g-(n-1) y_{I R}(g)}{n} .
\end{aligned}
$$

By the theorem of the maximum, we therefore have $v_{I R}^{\prime}(g)=\frac{1}{n}+\frac{n-1}{n} y_{I R}^{\prime}(g)$. Combining this expression with the first-order condition of (A2), we obtain

$$
y_{I R}^{\prime}(g)=\frac{1-n\left(1-\frac{\alpha}{2 \sqrt{g}}\right) / \delta}{1-n}
$$

for any $g<y_{I R}^{*}$. At $y_{I R}^{*}$, the left derivative must therefore be $y_{I R}^{-}\left(y_{I R}^{*}\right)$ $=\frac{1-n\left(1-\frac{\alpha}{2 \sqrt{y_{I R}^{*}}}\right) / \delta}{1-n}$. Imposing $y_{I R}^{-}\left(y_{I R}^{*}\right)=y_{I R}^{+}\left(y_{I R}^{*}\right)=1$, we obtain that in any concave Markov equilibrium with irreversibility we must have $y_{I R}^{*}=\left(\frac{\alpha}{2(1-\delta)}\right)^{2}$ as claimed.

\section{Proof of Proposition 3}

The efficient outcome (the social planner solution characterized in Section IA) can be sustained in the voluntary contribution game with reversible investment, when agents use nonstationary strategies entailing reversal to the unique concave Markov equilibrium characterized in Section IB. To show this, we construct strategies whose outcome is the efficient level of public good and we show that there is no profitable deviation from the equilibrium path. The symmetric strategy for each group member is to invest $i_{P}^{*}(g)=\min \left\{\frac{W}{n}, \frac{y_{P}^{*}-g}{n}\right\}$ if $g_{t}=y^{*}\left(g_{t-1}\right)$ (i.e., if the observed level of the public good at the beginning of the period is consistent with equilibrium strategies, or, in other words, it is the efficient level of public good given the stock of $g$ at the beginning of the previous period) and to invest $i_{R}^{*}(g)=\min \left\{\frac{W}{n}, \frac{y_{R}^{*}-g}{n}\right\}$, where $y_{R}^{*}<y_{P}^{*}$ (i.e., the investment associated with the Markov equilibrium characterized in Proposition 1) if $g_{t} \neq y^{*}\left(g_{t-1}\right)$ (i.e., if a deviation from equilibrium has occurred in the previous period). To prove that this strategy profile is an equilibrium we show that agents have no profitable deviation.

An agent's payoff if she follows the equilibrium strategy is

$$
\frac{W}{n}-i_{P}^{*}(g)+\alpha \sqrt{g+n i_{P}^{*}(g)}+\delta V_{E Q}\left(g+n i_{P}^{*}(g)\right) .
$$

An agent's payoff if she deviates (according to her most profitable deviation) is

$$
\frac{W}{n}+\frac{g}{n}+\alpha \sqrt{g-\frac{g}{n}+(n-1) i_{P}^{*}(g)}+\delta V_{D E V}\left(g-\frac{g}{n}+(n-1) i_{P}^{*}(g)\right) .
$$

An agent's most profitable deviation is to invest $-g / n$ (i.e., to subtract from the public good her share and to consume it). The gains from this deviation are greater 
the closer $g$ is to $y_{P}^{*}$. Therefore, we will check whether an agent has an incentive to deviate when $g \in\left[g_{P}, y_{P}^{*}\right]$, or whether

$$
\begin{aligned}
\frac{W}{n}-\frac{y_{P}^{*}-g}{n}+\alpha \sqrt{y_{P}^{*}}+\delta V_{E Q}\left(y_{P}^{*}\right) \geq & \frac{W}{n}+\frac{g}{n}+\alpha \sqrt{g-\frac{g}{n}+(n-1) \frac{y_{P}^{*}-g}{n}} \\
& +\delta V_{D E V}\left(g-\frac{g}{n}+(n-1) \frac{y_{P}^{*}-g}{n}\right),
\end{aligned}
$$

where $V_{E Q}\left(y_{P}^{*}\right)=\frac{1}{1-\delta}\left[\frac{W}{n}+\alpha \sqrt{y_{P}^{*}}\right]$, and

$$
\begin{aligned}
V_{D E V}\left(\frac{n-1}{n} y_{P}^{*}\right) & =\frac{W}{n}-\frac{y_{R}^{*}-\frac{n-1}{n} y_{P}^{*}}{n}+\alpha \sqrt{y_{R}^{*}}+\delta V_{D E V}\left(y_{R}^{*}\right) \\
& =\frac{W}{n}-\frac{y_{R}^{*}-\frac{n-1}{n} y_{P}^{*}}{n}+\alpha \sqrt{y_{R}^{*}}+\frac{\delta}{1-\delta}\left(\frac{W}{n}+\alpha \sqrt{y_{R}^{*}}\right) .
\end{aligned}
$$

After we plug in $V_{E Q}\left(y_{P}^{*}\right)$ and $V_{D E V}\left(\frac{n-1}{n} y_{P}^{*}\right)$ and we re-arrange terms, the inequality above becomes

$$
\frac{1}{1-\delta}\left[\alpha \sqrt{y_{P}^{*}}-\delta \alpha \sqrt{y_{R}^{*}}\right]-\frac{\delta}{n}\left[\frac{(n-1)}{n} y_{P}^{*}-y_{R}^{*}\right] \geq \alpha \sqrt{\frac{n-1}{n} y_{P}^{*}}+\frac{y_{P}^{*}}{n}
$$

Replacing $y_{P}^{*}=\left(\frac{\alpha n}{2(1-\delta)}\right)^{2}$ and $y_{R}^{*}=\left(\frac{\alpha n}{2(n-\delta)}\right)^{2}$, the inequality we want to prove becomes

$$
\begin{aligned}
& \frac{1}{1-\delta}\left[\frac{\alpha^{2} n}{2(1-\delta)}-\frac{\delta \alpha^{2} n}{2(n-\delta)}\right]-\frac{\delta(n-1) \alpha^{2}}{4(1-\delta)^{2}}+\delta n\left(\frac{\alpha}{2(n-\delta)}\right)^{2} \\
& \quad \geq \frac{\alpha^{2} n}{2(1-\delta)} \sqrt{\frac{n-1}{n}}+\frac{\alpha^{2} n}{4(1-\delta)^{2}} .
\end{aligned}
$$

Multiplying both sides by $(1-\delta)^{2}$ and rearranging, we have

$$
\begin{aligned}
& \frac{\alpha^{2} n}{2}-\frac{\alpha^{2} n}{4}-\frac{\delta \alpha^{2}(n-1)}{4} \\
& \quad \geq(1-\delta) \frac{\alpha^{2} n}{2} \sqrt{\frac{n-1}{n}}-\delta n\left(\frac{\alpha(1-\delta)}{2(n-\delta)}\right)^{2}+\frac{(1-\delta) \delta \alpha^{2} n}{2(n-\delta)}
\end{aligned}
$$

There is $\hat{\delta}_{R}$, such that $\forall \delta>\hat{\delta}_{R}$ the inequality above holds. To see this note that as $\delta$ approaches one the RHS approaches zero, while the LHS is positive for any $\delta \in[0,1]$. 
Using the parameters and the utility function of our experiments, $\hat{\delta}_{R}=0.80$ for $n=3$ and $\hat{\delta}_{R}=0.86$ for $n=5$. We use $\delta=0.75$, which means that, in the experimental setting, the efficient level of the public good cannot be sustained in equilibrium. However, it can be shown that nonstationary strategies of the type proposed above can sustain an almost efficient level of the public good, $y^{*}$. In this case, the inequality we want to prove is

$$
\frac{1}{1-0.75}\left[4 \sqrt{y^{*}}-3 \sqrt{y_{R}^{*}}\right]-\frac{0.75}{n}\left[\frac{(n-1)}{n} y^{*}-y_{R}^{*}\right] \geq 4 \sqrt{\frac{n-1}{n} y^{*}}+\frac{y^{*}}{n} .
$$

This inequality holds for $y^{*}=520$ in the treatment with 3 agents (where $\left.y_{P}^{*}=576\right)$ and for $y^{*}=1,332$ in the treatment with 5 agents (where $\left.y_{P}^{*}=1,600\right)$.

\section{Appendix B: AdDitional TABLES}

Table B1-Summary Statistics of Public Good Stock Per Period, RIE

\begin{tabular}{|c|c|c|c|c|c|c|c|c|}
\hline \multirow[b]{2}{*}{ Period } & \multicolumn{4}{|c|}{ RIE 3} & \multicolumn{4}{|c|}{ RIE 5} \\
\hline & Theory & Observations & Average & Mdn & Theory & Observations & Average & Mdn \\
\hline 1 & 7.11 & 110 & 29.24 & 29 & 5.54 & 60 & 47.38 & 50.5 \\
\hline 2 & 7.11 & 70 & 42.33 & 39.5 & 5.54 & 48 & 70.21 & 67 \\
\hline 3 & 7.11 & 51 & 33.02 & 20 & 5.54 & 33 & 59.97 & 34 \\
\hline 4 & 7.11 & 40 & 31.70 & 17.5 & 5.54 & 24 & 51.08 & 22.5 \\
\hline 5 & 7.11 & 33 & 29.85 & 11 & 5.54 & 21 & 42.29 & 27 \\
\hline 6 & 7.11 & 22 & 33.86 & 17.5 & 5.54 & 15 & 50.60 & 32 \\
\hline 7 & 7.11 & 11 & 15.55 & 12 & 5.54 & 12 & 36.58 & 36.5 \\
\hline 8 & 7.11 & 11 & 13.64 & 11 & 5.54 & 12 & 44.50 & 42.5 \\
\hline 9 & 7.11 & 11 & 15.82 & 16 & 5.54 & 6 & 32.00 & 35.5 \\
\hline 10 & 7.11 & 3 & 16.00 & 10 & 5.54 & 6 & 28.83 & 22 \\
\hline 11 & 7.11 & 3 & 21.67 & 29 & 5.54 & 3 & 24.00 & 24 \\
\hline 12 & 7.11 & 3 & 26.67 & 31 & 5.54 & 3 & 30.67 & 43 \\
\hline 13 & 7.11 & 3 & 30.33 & 31 & 5.54 & 3 & 31.67 & 37 \\
\hline
\end{tabular}

Note: Observations are groups.

Table B2-Summary Statistics of Public Good Stock Per Period, IIE

\begin{tabular}{|c|c|c|c|c|c|c|c|c|}
\hline \multirow[b]{2}{*}{ Period } & \multicolumn{4}{|c|}{ IIE 3} & \multicolumn{4}{|c|}{ IIE 5} \\
\hline & Theory & Observations & Average & Mdn & Theory & Observations & Average & Mdn \\
\hline 1 & 21.32 & 120 & 39.21 & 45 & 18.88 & 60 & 56.12 & 57 \\
\hline 2 & 27.04 & 92 & 83.35 & 92 & 24.56 & 57 & 109.86 & 114 \\
\hline 3 & 30.96 & 72 & 128.44 & 140.5 & 28.52 & 54 & 158.74 & 168.5 \\
\hline 4 & 33.92 & 68 & 162.90 & 177.5 & 31.64 & 42 & 200.36 & 218 \\
\hline 5 & 36.28 & 64 & 193.09 & 202.5 & 34.12 & 33 & 239.15 & 244 \\
\hline 6 & 38.24 & 56 & 233.86 & 238 & 36.24 & 30 & 270.67 & 270 \\
\hline 7 & 39.88 & 44 & 261.30 & 265.5 & 38.04 & 21 & 301.71 & 292 \\
\hline 8 & 41.32 & 36 & 291.72 & 287.5 & 39.64 & 18 & 323.93 & 324 \\
\hline 9 & 42.56 & 28 & 308.50 & 314.5 & 41.04 & 15 & 345.27 & 346 \\
\hline 10 & 43.64 & 20 & 298.40 & 293.5 & 42.28 & 12 & 386.08 & 367 \\
\hline 11 & 44.60 & 20 & 315.40 & 318 & 43.40 & 6 & 366.33 & 308.5 \\
\hline 12 & 45.48 & 16 & 303.75 & 291 & 44.44 & - & - & - \\
\hline 13 & 46.28 & 8 & 382.25 & 385 & 45.40 & - & - & - \\
\hline 14 & 47.00 & 8 & 400.38 & 391.5 & 46.28 & - & - & - \\
\hline 15 & 47.64 & 4 & 435.00 & 424.5 & 47.08 & - & - & - \\
\hline 16 & 48.24 & 4 & 465.00 & 459.5 & 47.84 & - & - & - \\
\hline 17 & 48.80 & 4 & 495.75 & 496 & 48.52 & - & - & - \\
\hline
\end{tabular}

Note: Observations are groups. 
Table B3-Summary Statistics of Individual Investment Per Period, RIE

\begin{tabular}{|c|c|c|c|c|c|c|c|c|}
\hline \multirow[b]{2}{*}{ Period } & \multicolumn{4}{|c|}{ RIE 3} & \multicolumn{4}{|c|}{ RIE 5} \\
\hline & Observations & Average & Mdn & SD & Observations & Average & Mdn & SD \\
\hline 1 & 330 & 9.75 & 10 & 6.27 & 300 & 9.48 & 11 & 6.76 \\
\hline 2 & 210 & 4.45 & 5 & 8.97 & 240 & 3.79 & 4 & 10.49 \\
\hline 3 & 153 & -2.85 & -3 & 10.72 & 165 & -1.78 & -2 & 11.61 \\
\hline 4 & 120 & -1.13 & 0 & 9.62 & 120 & -1.74 & 0 & 12.28 \\
\hline 5 & 99 & -0.61 & 0 & 7.86 & 105 & -2.8 & -2 & 11.29 \\
\hline 6 & 66 & 2.02 & 1 & 6.08 & 75 & 0.76 & 0 & 11.64 \\
\hline 7 & 33 & 0.55 & 0 & 4.85 & 60 & -2.37 & -1.5 & 12.87 \\
\hline 8 & 33 & -0.64 & 0 & 4.33 & 60 & 1.58 & 0 & 9.45 \\
\hline 9 & 33 & 0.73 & 0 & 5.85 & 30 & -1.93 & -2 & 9.09 \\
\hline 10 & 9 & -3.22 & -5 & 6.34 & 30 & -0.63 & -2 & 8.03 \\
\hline 11 & 9 & 1.89 & 4 & 6.15 & 15 & 3.33 & 2 & 5.37 \\
\hline 12 & 9 & 1.67 & 3 & 9.92 & 15 & 1.33 & -1 & 6.38 \\
\hline 13 & 9 & 1.22 & 0 & 9.09 & 15 & 0.20 & 0 & 6.68 \\
\hline
\end{tabular}

Note: Observations are individual investment decisions.

Table B4-Summary Statistics of Individual Investment Per Period, IIE

\begin{tabular}{|c|c|c|c|c|c|c|c|c|}
\hline \multirow[b]{2}{*}{ Period } & \multicolumn{4}{|c|}{ IIE 3} & \multicolumn{4}{|c|}{ IIE 5} \\
\hline & Observations & Average & Mdn & SD & Observations & Average & Mdn & SD \\
\hline 1 & 360 & 13.07 & 15 & 6.87 & 300 & 11.22 & 14 & 5.36 \\
\hline 2 & 276 & 13.54 & 15 & 6.65 & 285 & 10.68 & 12 & 5.63 \\
\hline 3 & 216 & 13.36 & 15 & 6.44 & 270 & 9.88 & 11 & 5.97 \\
\hline 4 & 204 & 12.00 & 15 & 6.88 & 210 & 8.83 & 8.5 & 6.23 \\
\hline 5 & 192 & 10.17 & 10 & 7.46 & 165 & 7.73 & 8 & 6.28 \\
\hline 6 & 168 & 9.51 & 10 & 7.63 & 150 & 6.70 & 5 & 6.29 \\
\hline 7 & 132 & 8.28 & 5 & 7.80 & 105 & 6.43 & 4 & 6.51 \\
\hline 8 & 108 & 6.39 & 5 & 6.90 & 90 & 5.91 & 3 & 6.32 \\
\hline 9 & 84 & 5.77 & 4.5 & 6.85 & 75 & 5.27 & 2 & 6.33 \\
\hline 10 & 60 & 5.87 & 4.5 & 6.67 & 60 & 4.00 & 0 & 6.11 \\
\hline 11 & 60 & 5.67 & 1.5 & 7.27 & 30 & 2.73 & 0 & 5.07 \\
\hline 12 & 48 & 4.15 & 1 & 6.15 & - & - & - & - \\
\hline 13 & 24 & 6.21 & 4.5 & 7.42 & - & - & - & - \\
\hline 14 & 24 & 6.04 & 3 & 7.62 & - & - & - & - \\
\hline 15 & 12 & 10.83 & 10 & 8.75 & - & - & - & - \\
\hline 16 & 12 & 10.00 & 8 & 7.89 & - & - & - & - \\
\hline 17 & 12 & 10.25 & 9 & 7.69 & - & - & - & - \\
\hline
\end{tabular}

Note: Observations are individual investment decisions.

Table B5- $p$-Values of $t$-Tests on the Equality of Public Good Stock Averages

\begin{tabular}{lcccc}
\hline \hline Period & RIE3/IIE3 & RIE5/IIE5 & RIE3/RIE5 & IIE3/IIE5 \\
\hline 1 & 0.000 & 0.005 & 0.000 & 0.000 \\
2 & 0.000 & 0.000 & 0.000 & 0.000 \\
3 & 0.000 & 0.000 & 0.023 & 0.000 \\
4 & 0.000 & 0.000 & 0.150 & 0.002 \\
5 & 0.000 & 0.000 & 0.402 & 0.013 \\
6 & 0.000 & 0.000 & 0.381 & 0.120 \\
7 & 0.000 & 0.000 & 0.043 & 0.223 \\
8 & 0.000 & 0.000 & 0.006 & 0.418 \\
9 & 0.000 & 0.000 & 0.070 & 0.478 \\
10 & 0.000 & 0.000 & 0.326 & 0.154 \\
\hline
\end{tabular}


Table B6- $p$-Values of Wilcoxon-Mann-Whitney Tests on Stocks, Early versus LATE Matches

\begin{tabular}{lcccc}
\hline \hline Period & RIE3 & RIE5 & IIE3 & IIE5 \\
\hline 1 & 0.138 & 0.611 & 0.096 & 0.004 \\
2 & 0.168 & 0.161 & 0.000 & 0.010 \\
3 & 0.009 & 0.591 & 0.000 & 0.008 \\
4 & 0.072 & 0.057 & 0.000 & 0.277 \\
5 & 0.916 & 0.904 & 0.000 & 0.590 \\
6 & 0.116 & 1.000 & 0.000 & 0.645 \\
7 & 0.658 & 0.400 & 0.002 & 0.593 \\
8 & 0.215 & 0.941 & 0.011 & 0.592 \\
9 & 0.086 & - & 0.002 & 0.574 \\
10 & - & - & 0.198 & 0.920 \\
\hline
\end{tabular}

Notes: Early matches are one through five. Late matches are six through ten. We lack observations in both groups for period ten in RIE3 and for periods nine and ten in RIE5.

\section{Appendix C: Sample Experimental Instructions (RIE5)}

Thank you for agreeing to participate in this experiment. During the experiment we require your complete, undistracted attention and ask that you follow instructions carefully. Please turn off your cell phones. Do not open other applications on your computer, chat with other students, or engage in other distracting activities, such as reading books, doing homework, etc. You will be paid for your participation in cash, at the end of the experiment. Different participants may earn different amounts. What you earn depends partly on your decisions, partly on the decisions of others, and partly on chance. It is important that you not talk or in any way try to communicate with other participants during the experiments.

Following the instructions, there will be a practice session and a short comprehension quiz. All questions on the quiz must be answered correctly before continuing to the paid session. At the end you will be paid in private and you are under no obligation to tell others how much you earned. Your earnings are denominated in FRANCS which will be converted to dollars at the rate of 75 FRANCS to a DOLLAR.

This is an experiment in group decision making. The experiment will take place over a sequence of ten matches. We begin the match by dividing you into THREE groups of five members each. Each of you is assigned to exactly one of these groups. In each match each member of your group will make investment decisions.

In each round, each member of your group has a budget of 16 francs. Each member must individually decide how to divide his or her budget into private investment and project investment, in integer amounts. The private investment always has to be greater than or equal than zero. The project investment can be either positive, or zero, or negative. Any amount you allocate to private investment goes directly to your earnings for this round. The project investment produces earnings for all group members in the following way.

\section{[SHOW SLIDE]}

The project earnings in a round depend on the size of the project at the end of that round. Specifically, each group member earns an amount in francs proportional to 
the square root of the size of the project at the end of the round (precisely equal to $4 \times \operatorname{sqrt}($ project size)). Thus, for example, if the size of the project at the end of the round equals 9 , then each member earns exactly $4 \times \operatorname{sqrt}(9)=12$ additional francs in that round. If the size is equal to 36 , each member earns exactly $4 \times \operatorname{sqrt}(36)=24$ additional francs in that round. In your display, earnings are always rounded to two decimal places. So, for example if the project size at the end of a round equals 5, each member earns $4 \times \operatorname{sqrt}(5)=8.94$ francs from the project in that round.

The second important fact about the project is that it is durable. That is, project investment in a round increases or decreases the size not just for that round, but also for all future rounds. The size of your group's project starts at zero in the first round of the match. At the end of the first round it is equal to the sum of your group members' project investment in that round. This amount gets carried over to the second round. Whenever the size of the project is greater than zero, you can propose a negative project investment. However, in this case, the proposed negative investment cannot exceed one-fifth of the size of the project at the beginning of the round (in other words, you can dispose only of your share of the project). At the end of the second round, the size of the project equals to the combined amount invested in the project in rounds 1 and 2 by all members of your group, and so forth. So, every round project investment changes the size of the project for the current round and all future rounds of the match.

The total number of rounds in a match will depend on the rolling of a fair eight-sided die. When the first round ends, we roll it to decide whether to move on to the second round. If the die comes up a one or a two we do not go on to round 2, and the match is over. Otherwise, we continue to the next round. We continue to more rounds, until a one or a two is rolled at the end of a round and the match ends. At the end of each round your earnings for that round are computed by adding the project earnings to your private investment. For example, if your private investment is 20 and the end-of-round project size is 9, then your earnings for that round equal $20+4 \times$ $\operatorname{sqrt}(9)=20+12=32$. Your earnings for the match equal the sum of the earnings in all rounds of that match.

After the first match ends, we move to match 2. In this new match, you are reshuffled randomly into THREE new groups of five members each. The project size in your new group again starts out at zero. The match then proceeds the same way as match 1. After match 10, the experiment is over. Your total earnings for the experiment are the sum of your earnings over all rounds and all matches.

We will now go through one practice match very slowly. During the practice match, please do not hit any keys until I tell you, and when you are prompted by the computer to enter information, please wait for me to tell you exactly what to enter. You are not paid for this practice match.

\section{[AUTHENTICATE CLIENTS]}

Please double click on the icon on your desktop that says BP2. When the computer prompts you for your name, type your First and Last name. Then click SUBMIT and wait for further instructions. You now see the first screen of the experiment on your computer. It should look similar to this screen. 


\section{[SHOW SLIDE]}

At the top left of the screen, you see your subject ID. In the top right you can see that you have been assigned by the computer to a group of FIVE subjects, and assigned a group member number: 1, 2, 3, 4, or 5. This group assignment and your member number stays the same for all rounds of this match, but will change across matches. It is very important that you take careful note of your group member number.

As a visual aid, there is a graph on the left that shows exactly how project earnings will depend on project size. The current size of the project is marked with a large dot at the origin. If each member of your group decides to invest nothing this period, then this will be the size that determines your project earnings at the end of the round. You can use your mouse to move the curser along the curve to figure out what your earnings will be for different levels of project investment. Also, if you type an amount in the Project Investment box, the computer will compute and display the corresponding project earnings for you just below the box. Take a minute to practice using your curser to move along the curve, and typing in different possible investment levels. But do not hit the confirm button yet.

At this time, go ahead and type in any investment decision you wish and hit the confirm button. You are not paid for this practice match so it does not matter what you enter.

\section{[SHOW SLIDE]}

This screen now summarizes the outcome of the round. Here you see your group member number, and the end of round project size. The investment decisions of each member are displayed in a table. Below the table are displayed your earnings for the round, given the outcome. This marks the end of the round. The table with columns in the bottom of your screen is the History panel and summarizes all of this important information.

We now roll an eight-sided die to decide whether to move on to round 2. If the die comes up a one or a two, we do not go on to round 2, and the match is over. If the die comes up three through eight, we continue to a second round of the match. [Roll die and do second round unless it comes up a one or two. Next say "the die roll was X, so we will continue to the next round." If X = (one or two) say "if this was a real match, there would be no second round. That would be the end of the match. However, we want to go through one more practice round to make sure you are familiar with the computer interface."]

\section{[SHOW SLIDE]}

In this second round, you keep the same group member number as in the first round, and the members of your group all stay the same. Notice that the project investment from round 1 carries over, so the round 2 beginning project size equals the project size at the end of round 1. In this second round please follow the same instructions of the first round. You can go ahead now. Since this is a practice match, 
we will not roll a die after the second round, and the practice match will end. During the paid matches, each match will continue until the die comes up a one or a two.

\section{[DISPLAY SUMMARY SCREEN]}

Now we are ready for the comprehension quiz. Everyone must answer all the questions correctly before we go to the paid matches. The quiz has two pages. You must answer all the questions on Page 1 of the quiz to proceed to Page 2. If you answer any of the questions on a page incorrectly, you will be asked to try again. Please raise your hand if you have any questions during the quiz, and we will come to your desk and answer your question in private.

\section{[REASSURE THEM IT'S OK TO ASK FOR HELP] [WAIT FOR END OF QUIZ]}

Are there any questions before we begin with the paid session?

\section{[WAIT FOR QUESTIONS]}

We will now begin with the first of ten paid matches of the experiment. Please pull out your dividers for the paid session of the experiment. If there are any problems or questions from this point on, raise your hand and an experimenter will come and assist you in private.

\section{REFERENCES}

Andreoni, James, and Rachel Croson. 2008. "Partner versus Strangers: The Effect of Random Rematching in Public Goods Experiments." In Handbook of Experimental Economics Results, Vol. 1, edited by Charles Plott and Vernon Smith, 776-83. Amsterdam: North-Holland.

Battaglini, Marco, Salvatore Nunnari, and Thomas R. Palfrey. 2010. "Political Institutions and the Dynamics of Public Investment." California Institute of Technology Social Science Working Paper 1318.

Battaglini, Marco, Salvatore Nunnari, and Thomas R. Palfrey. 2012. "Legislative Bargaining and the Dynamics of Public Investment." American Political Science Review 106 (2): 407-29.

Battaglini, Marco, Salvatore Nunnari, and Thomas R. Palfrey. 2014. "Dynamic Free Riding with Irreversible Investments.” American Economic Review 104 (9): 2858-71.

Battaglini, Marco, Salvatore Nunnari, and Thomas R. Palfrey. 2016. "The Dynamic Free Rider Problem: A Laboratory Study: Dataset.” American Economic Journal: Microeconomics. http://dx.doi. org/10.1257/mic.20150126.

Battaglini, Marco, and Thomas R. Palfrey. 2012. “The Dynamics of Distributive Politics.” Economic Theory 49 (3): 739-77.

Burlando, Roberto M., and Francesco Guala. 2005. "Heterogeneous Agents in Public Goods Experiments." Experimental Economics 8 (1): 35-54.

Chaudhuri, Ananish. 2011. "Sustaining cooperation in laboratory public goods experiments: A selective survey of the literature." Experimental Economics 14 (1): 47-83.

Choi, Syngjoo, Douglas Gale, and Shachar Kariv. 2008. "Sequential equilibrium in monotone games: A theory-based analysis of experimental data." Journal of Economic Theory 143 (1): 302-30.

Choi, Syngjoo, Douglas Gale, Shachar Kariv, and Thomas Palfrey. 2011. "Network architecture, salience and coordination." Games and Economic Behavior 73 (1): 76-90.

Cooper, David J., and Kai-Uwe Kühn. 2014. "Communication, Renegotiation, and the Scope for Collusion." American Economic Journal: Microeconomics 6 (2): 247-78.

Dal Bó, Pedro, and Guillaume Fréchette. 2014. "On the Determinants of Cooperation in Infinitely Repeated Games: A Survey.” http://isites.harvard.edu/fs/docs/icb.topic1516259.files/ DalBoFrechette_JEL.pdf. 
Diev, Pavel, and Walid Hichri. 2008. "Dynamic voluntary contributions to a discrete public good: Experimental evidence." Economics Bulletin 3 (23).

Dockner, Engelbert J., and Ngo Van Long. 1993. "International Pollution Control: Cooperative versus Noncooperative Strategies." Journal of Environmental Economics and Management 25 (1): 13-29.

Dorsey, Robert E. 1992. "The voluntary contributions mechanism with real time revisions." Public Choice 73 (3): 261-82.

Duffy, John, Jack Ochs, and Lisa Vesterlund. 2007. "Giving little by little: Dynamic voluntary contribution games." Journal of Public Economics 91 (9): 1708-30.

-Fershtman, Chaim, and Shmuel Nitzan. 1991. "Dynamic voluntary provision of public goods." European Economic Review 35 (5): 1057-67.

-Fischbacher, Urs, and Simon Gächter. 2010. "Social Preferences, Beliefs, and the Dynamics of Free Riding in Public Goods Experiments." American Economic Review 100 (1): 541-56.

- Fischbacher, Urs, Simon Gächter, and Ernst Fehr. 2001. "Are people conditionally cooperative? Evidence from a public goods experiment.” Economics Letters 71 (3): 397-404.

- Harrison, Glenn W., and Jack Hirschleifer. 1989. "An Experimental Evaluation of Weakest Link/Best Shot Models of Public Goods.” Journal of Political Economy 97 (1): 201-25.

-Herr, Andrew, Roy Gardner, and James M. Walker. 1997. "An Experimental Study of Time-Independent and Time-Dependent Externalities in the Commons." Games and Economic Behavior 19 (1): 77-96.

-Isaac, R. Mark, James M. Walker, and Susan H. Thomas. 1984. "Divergent evidence on free riding: An experimental examination of possible explanations." Public Choice 43 (2): 113-49.

-Keser, Claudia, and Frans Van Winden. 2000. "Conditional Cooperation and Voluntary Contributions to Public Goods." Scandinavian Journal of Economics 102 (1): 23-39.

Laury, Susan K., and Charles A. Holt. 2008. "Voluntary Provision of Public Goods: Experimental Results with Interior Nash Equilibria." In Handbook of Experimental Economics Results, Vol. 1, edited by Charles R. Plott and Vernon L. Smith, 792-801. Amsterdam: North-Holland.

Ledyard, John. 1995. "Public Goods: A Survey of Experimental Research." In Handbook of Experimental Economics, edited by John H. Kagel and Alvin E. Roth, 111-94. Princeton: Princeton University Press.

- Lei, Vivian, and Charles N. Noussair. 2002. "An Experimental Test of an Optimal Growth Model." American Economic Review 92 (3): 549-70.

-Marx, Leslie M., and Steven A. Matthews. 2000. "Dynamic Voluntary Contribution to a Public Project." Review of Economic Studies 67 (2): 327-58.

-Matthews, Steven A. 2013. "Achievable outcomes of dynamic contribution games." Theoretical Economics 8 (2): 365-403.

Noussair, Charles, and Kenneth Matheny. 2000. "An experimental study of decisions in dynamic optimization problems." Economic Theory 15 (2): 389-419.

- Noussair, Charles, and Cindy Soo. 2008. "Voluntary contributions to a dynamic public good: Experimental evidence." Economics Letters 98 (1): 71-77.

-Ostrom, Elinor. 1999. "Coping with the Tragedies of the Commons." Annual Review of Political Science 2: 493-535.

Palfrey, Thomas R., and Howard Rosenthal. 1991. "Testing Game-Theoretic Models of Free Riding: New Evidence on Probability Bias and Learning." In Laboratory Research in Political Economy, edited by Thomas R. Palfrey, 239-68. Ann Arbor: University of Michigan Press.

Pevnitskaya, Svetlana, and Dmitry Ryvkin. 2013. "Environmental context and termination uncertainty in games with a dynamic public bad." Environmental and Development Economics 18 (1): $27-49$.

Saijo, Tatsuyoshi, Katerina Sherstyuk, Nori Tarui, and Majah-Leah Ravago. 2009. "Games with Dynamic Externalities and Climate Change Experiments." http://www.msri.org/attachments/ workshops/515/climate-change_draft050209.pdf.

Samuelson, Paul A. 1954. "The Pure Theory of Public Expenditure." Review of Economics and Statistics 36 (4): 387-89.

-Silvestre, Joaquim. 2003. "Wicksell, Lindahl and the Theory of Public Goods." Scandinavian Journal of Economics 105 (4): 527-53.

Vespa, Emanuel. 2016. "An Experimental Investigation of Strategies in Dynamic Games.” University of California, Santa Barbara Working Paper.

Vesterlund, Lise. 2016. "Using Experimental Methods to Understand Why and How We Give to Charity." In Handbook of Experimental Economics, Vol. 2, edited by John H. Kagel and Alvin E. Roth, 91-151. Princeton: Princeton University Press. 